\title{
Pair correlation of sums of rationals with bounded height
}

\author{
By Emre Alkan at Istanbul, Maosheng Xiong at University Park, \\ and Alexandru Zaharescu at Bucharest and Urbana
}

\begin{abstract}
For each positive integer $Q$, let $\mathscr{F}_{Q}$ denote the Farey sequence of order $Q$. We prove the existence of the pair correlation measure associated to the sum $\mathscr{F}_{Q}+\mathscr{F}_{Q}$ modulo 1, as $Q$ tends to infinity, and compute the corresponding limiting pair correlation function.
\end{abstract}

\section{Introduction}

The study of local spacings, which measure the distribution of a sequence in a more subtle way than the classical Weyl uniform distribution ([46]), was initiated by physicists (see Wigner [47] and Dyson [11], [12], [13]), in order to understand the spectra of high energies. These notions have received a great deal of attention in many areas of mathematical physics, analysis, probability theory and number theory. In most cases of interest in number theory it is very challenging to prove the existence of the limiting spacing measures. Many such sequences are predicted to have a Poisson distribution, and some important results of this type are due to Hooley [19], [20] on residue classes relatively prime with a large modulus $q$, Gallagher [17] on gaps between primes, Sarnak [39] on values at integers of binary quadratic forms, and Rudnick and Sarnak on pair correlation of fractional parts of polynomials [34]. Further results have been obtained by a number of authors. Primitive roots modulo $p$ were studied in [9], and the distribution of visible points from the origin in dilations of a region $\Omega$ was established in [4]. The spacing distribution of fractional parts of lacunary sequences has been obtained by Rudnick and one of the authors in [36] and [38] (see also [7]), and the distribution of small powers of a primitive root was studied in [37]. Boca and one of the authors [6] investigated the pair correlation of values of rational functions modulo p. Kurlberg and Rudnick [27] (see also [26]) established the distribution of squares modulo highly composite integers. The spacings between the energy levels of a two-dimensional harmonic oscillator (see Pandey, Bohigas and Giannoni [32] and Bleher

First author is supported in part by TUBITAK Career Award and Distinguished Young Scholar Award, TUBA-GEBIP of Turkish Academy of Sciences. Third author is supported in part by National Science Foundation Grant DMS-0456615. 
[2], [3]) are essentially those between the numbers $\alpha n(\bmod 1)$, where the gaps take at most three values (see Sós [40] and Swierczkowski [41]). The distribution of energy levels of a boxed oscillator reduces to that of $\alpha n^{2}(\bmod 1)$, which is conjectured to be Poissonian (see Berry and Tabor [1]). Rudnick, Sarnak and one of the authors [35] (see also [50]) proved that this conjecture holds true for a large class of numbers $\alpha$ satisfying certain Diophantine conditions. Eigenvalues on multidimensional flat tori, and values at integers of homogeneous polynomials, were studied by Vanderkam [42], [43], [44]. Correlation densities of inhomogeneous quadratic forms were investigated by Marklof [28], [29]. The distribution of the fractional part of $\sqrt{n}$ was established by Elkies and McMullen [14]. The distribution of imaginary parts of zeros of primitive L-functions is believed to be the same as the GUE distribution studied by Random Matrix Theory. Important work in this area was done by Montgomery [30], Rudnick and Sarnak [33], and Katz and Sarnak [24], see also [25]. One striking difference between the GUE model and the Poissonian model is that the density function vanishes at the origin in the GUE case but not in the Poissonian case. For this reason, it is said that in the Poissonian case one has "level clustering" while in the GUE case one has "level repulsion". Here the word "level", coined by physicists, refers to the possibly infinitely many stages of a process. One has an even stronger repulsion in the context described below.

Here we investigate a new type of question, which concerns two different notions: the pair correlation of the given sequence and the natural additive structure of the ambient space. More specifically, for each positive integer $Q$ let $\mathscr{F}_{Q}$ denote the Farey sequence of order $Q$ (for basic properties of the Farey sequence see [18]), as the $Q$ th level of our process, that is, the set of all rationals in $[0,1]$ of height bounded by $Q$ (the height of a rational number, in irreducible form, is defined to be the maximum of the absolute values of its numerator and denominator). The pair correlation measure associated to $\mathscr{F}_{Q}$ was proved to converge, as $Q \rightarrow \infty$, by Boca and one of the authors [8]. They showed that the limiting measure is absolutely continuous with respect to the Lebesgue measure, and provided an explicit formula for the corresponding limiting pair correlation function $g(\lambda)$,

$$
g(\lambda)=\frac{6}{\pi^{2} \lambda^{2}} \sum_{1 \leqq k \leqq \frac{\pi^{2} \lambda}{3}} \varphi(k) \log \frac{\pi^{2} \lambda}{3 k},
$$

for any $\lambda>0$, where $\varphi$ is Euler's totient function.

Let $\mathscr{F}_{Q}=\{a / b \mid 1 \leqq a \leqq b \leqq Q,(a, b)=1\}$ be the set of Farey fractions of order $Q$ and also let $\mathscr{F}_{Q}+\mathscr{F}_{Q} \subset[0,1)$ denote the set of all sums of pairs of fractions in $\mathscr{F}_{Q}$ written modulo 1. Our goal is to understand whether addition of Farey fractions influences the pair correlation measure. For this purpose, we compare the pair correlation of $\mathscr{F}_{Q}+\mathscr{F}_{Q}(\bmod 1)$ with that of $\mathscr{F}_{Q}$, as $Q \rightarrow \infty$. From a technical point of view the pair correlation measure of the sum $\mathscr{F}_{Q}+\mathscr{F}_{Q}$ is more difficult to handle than that of $\mathscr{F}_{Q}$. The Weil bounds [45], [15] for Kloosterman sums, which played a decisive role in [8], fail to solve the problem. A natural strategy would be to employ Deligne bounds [10] for two dimensional hyper-Kloosterman sums, but the range of the sums turns out to be too short for this method to succeed either. Karatsuba [21], [22], [23] devised a method for bounding certain exponential sums, and Friedlander and Iwaniec applied it successfully in [16], but our short ranges are outside the scope of this method either. As pointed out in [48], [49], one sometimes obtains more cancellation by averaging the pair correlations themselves rather than by averaging their expressions in terms of exponential sums. Inspired by this idea, we adjust 
our use of exponential sums, and barely obtain enough cancellation to complete the proof. In order to state our main result, we introduce a multiplicative arithmetic function $\psi$, which plays a similar role for $\mathscr{F}_{Q}+\mathscr{F}_{Q}$ to the one played by Euler's function for $\mathscr{F}_{Q}$. We define $\psi$ in terms of its associated Dirichlet series,

$$
\sum_{n=1}^{\infty} \frac{\psi(n)}{n^{s}}=\frac{\zeta(s-1)}{\zeta^{4}(s)} \prod_{p \text { prime }} H_{p}(s)
$$

where $\zeta(s)$ is the Riemann zeta function, and $H_{p}(s)$ is given by

$$
H_{p}(s)=1+\frac{(p-1)(p+4)}{p(p+3)}\left\{-\frac{4}{p-1}+\sum_{k=1}^{\infty} \frac{\left(1-p^{(k+1)(1-s)}\right)^{4}-\left(1-p^{k(1-s)}\right)^{4}}{\left(1-p^{1-s}\right) p^{k(2-s)}}\right\} .
$$

Theorem 1. The limiting pair correlation function of $\mathscr{F}_{Q}+\mathscr{F}_{Q}$ modulo 1 exists, as $Q \rightarrow \infty$, on any subinterval $\mathbf{I} \subset[0,1]$, and is given by

$$
g_{2}(\lambda)=\frac{c}{\pi^{2} \lambda^{2}} \sum_{1 \leqq k \leqq \frac{\pi^{4} \lambda}{9}} \psi(k) \log ^{3} \frac{\pi^{4} \lambda}{9 k}
$$

for any $\lambda>0$, where

$$
c=\prod_{p \text { prime }}\left(1-\frac{2}{p(p+1)}\right)\left(1-\frac{3}{p(p+2)}\right) .
$$

The above functions $g(\lambda)$ and $g_{2}(\lambda)$ being distinct, we see that addition of Farey fractions does influence, in this sense, the pair correlation. Their graphs are shown in Figure 1, together with $g_{\mathrm{GUE}}(\lambda)=1-\left(\frac{\sin \pi \lambda}{\pi \lambda}\right)^{2}$ and $g_{\text {Poisson }}=$ constant equal to 1 .

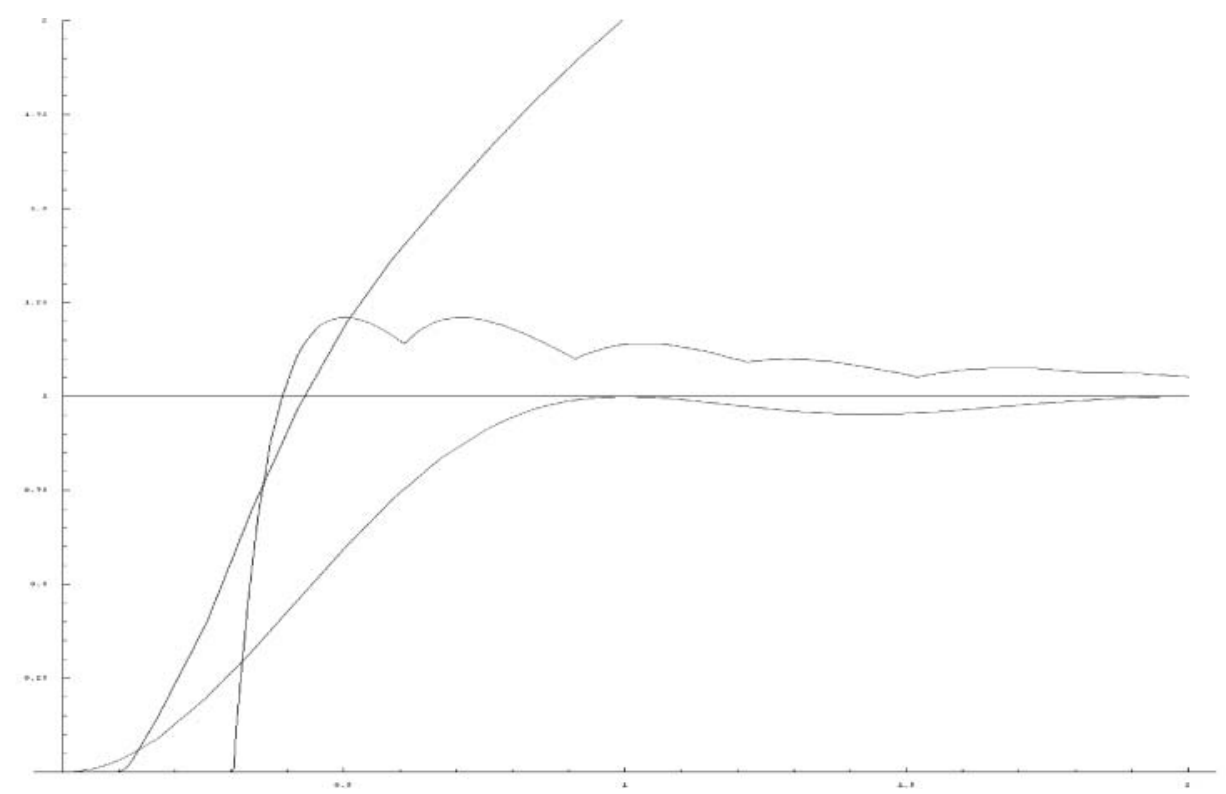

Figure 1. Graphs of $g(\lambda), g_{2}(\lambda), g_{\mathrm{GUE}}(\lambda)$ and $g_{\text {Poisson }}$ 
Acknowledgments. The authors are grateful to the referee for many valuable suggestions.

\section{A uniform distribution result}

Let $\mathscr{F}_{Q}=\left\{\gamma_{1}, \ldots, \gamma_{N(Q)}\right\}$ denote the Farey sequence of order $Q$ with $1 / Q=\gamma_{1}<\gamma_{2}<\cdots<\gamma_{N(Q)}=1$ and $\mathscr{F}=\left(\mathscr{F}_{Q}\right)_{Q}$. Let $x_{i j} \equiv \gamma_{i}+\gamma_{j}(\bmod 1)$ and denote by the set $\mathcal{G}_{Q}:=\left\{x_{i j} \mid 1 \leqq i, j \leqq N(Q)\right\}=\mathscr{F}_{Q}+\mathscr{F}_{Q}(\bmod 1)$ counting multiplicities. The sequence of sequences $\mathcal{G}=\left(\mathcal{G}_{Q}\right)_{Q \in \mathbb{N}}$ is uniformly distributed along the unit interval. More precisely,

Lemma 1. For any subinterval $\mathbf{I} \subset[0,1]$, denote $\mathcal{G}_{\mathbf{I}}(Q):=\mathcal{G}_{Q} \cap \mathbf{I}$. Then

$$
\# \mathcal{G}_{\mathbf{I}}(Q)=\frac{9|\mathbf{I}|}{\pi^{4}} Q^{4}+O\left(Q^{3}(\log Q)^{3 / 2}\right)
$$

Our method actually gives a more general counting result. For any continuously differentiable function $f: R^{k} \rightarrow R$ with compact support, we define

$$
D f=\left\langle\frac{\partial f}{\partial x_{1}}, \frac{\partial f}{\partial x_{2}}, \ldots, \frac{\partial f}{\partial x_{k}}\right\rangle
$$

and

$$
\|D f\|_{\infty}=\sum_{j=1}^{k}\left\|\frac{\partial f}{\partial x_{j}}\right\|_{\infty}
$$

Lemma 2. Suppose I is a finite interval, $G \in C^{1}(\mathbb{R})$ with $\operatorname{Supp}(G) \subset \mathbf{I}$. Define

$$
g(y)=\sum_{n \in \mathbb{Z}} G(y+n), \quad S_{Q, G}=\sum_{\gamma, \gamma^{\prime} \in \mathscr{F}_{Q}} g\left(\gamma+\gamma^{\prime}\right) .
$$

Then

$$
S_{Q, G}=\left(\int_{\mathbf{I}} G(x) \mathrm{d} x\right) \frac{9 Q^{4}}{\pi^{4}}+E_{Q, G}
$$

where

$$
E_{Q, G} \ll Q^{2}(\log Q)^{3}\|D G\|_{\infty}|\mathbf{I}|+Q^{3} \log Q\left|\int_{\mathbf{I}} G(x) \mathrm{d} x\right| .
$$

Proof of Lemma 2. For $y \in \mathbb{R}$, let

$$
g(y)=\sum_{n \in \mathbb{Z}} a_{n} e(n y)
$$

be the Fourier series expansion of $g$. Then 


$$
\begin{aligned}
S_{Q, G} & =\sum_{\gamma, \gamma^{\prime} \in \mathscr{F}_{Q}} \sum_{n \in \mathbb{Z}} a_{n} e\left(n\left(\gamma+\gamma^{\prime}\right)\right)=\sum_{n \in \mathbb{Z}} a_{n} \sum_{\gamma \in \mathscr{F}_{Q}} e(n \gamma) \sum_{\gamma^{\prime} \in \mathscr{F}_{Q}} e\left(n \gamma^{\prime}\right) \\
& \left.=\sum_{n \in \mathbb{Z}} a_{n} \sum_{\substack{1 \leqq d \leqq Q \\
d \mid n}} d M\left(\frac{Q}{d}\right)\right)^{2} \\
& =\sum_{1 \leqq d_{1}, d_{2} \leqq Q} d_{1} d_{2} M\left(\frac{Q}{d_{1}}\right) M\left(\frac{Q}{d_{2}}\right) \sum_{l \in \mathbb{Z}} a_{\left[d_{1}, d_{2}\right] l} .
\end{aligned}
$$

Consider for each $y>0$ the function

$$
G_{y}(x)=\frac{1}{y} G\left(\frac{x}{y}\right), \quad x \in \mathbb{R} .
$$

By properties of the Fourier transform,

$$
\hat{G}_{y}(x)=\hat{G}(y x)
$$

and using Poisson summation formula,

$$
\sum_{l \in \mathbb{Z}} a_{\left[d_{1}, d_{2}\right] l}=\sum_{l \in \mathbb{Z}} \hat{G}\left(\left[d_{1}, d_{2}\right] l\right)=\sum_{l \in \mathbb{Z}} \hat{G}_{\left[d_{1}, d_{2}\right]}(l)=\sum_{l \in \mathbb{Z}} G_{\left[d_{1}, d_{2}\right]}(l) .
$$

Thus

$$
S_{Q, G}=\sum_{1 \leqq d_{1}, d_{2} \leqq Q} d_{1} d_{2} M\left(\frac{Q}{d_{1}}\right) M\left(\frac{Q}{d_{2}}\right) \sum_{l \in \mathbb{Z}} \frac{1}{\left[d_{1}, d_{2}\right]} G\left(\frac{l}{\left[d_{1}, d_{2}\right]}\right) .
$$

Applying [5], Lemma 8, we obtain

$$
\left.\sum_{l \in \mathbb{Z}} \frac{1}{\left[d_{1}, d_{2}\right]} G\left(\frac{l}{\left[d_{1}, d_{2}\right]}\right)=\int_{\mathbf{I}} G(x) \mathrm{d} x+O\left(\|D G\|_{\infty} \frac{|\mathbf{I}|}{\left[d_{1}, d_{2}\right]}+\frac{2}{\left[d_{1}, d_{2}\right]^{2}}\right)\right) .
$$

Therefore,

$$
\begin{aligned}
S_{Q, G} & =\left(\int_{\mathbf{I}} G(x) \mathrm{d} x\right)\left(\sum_{1 \leqq d_{1}, d_{2} \leqq Q} d_{1} d_{2} M\left(\frac{Q}{d_{1}}\right) M\left(\frac{Q}{d_{2}}\right)\right)+E_{G, 1} \\
& =\left(\int_{\mathbf{I}} G(x) \mathrm{d} x\right)\left(\sum_{1 \leqq d \leqq Q} d M\left(\frac{Q}{d}\right)\right)^{2}+E_{G, 1}
\end{aligned}
$$

where

$$
\left.E_{G, 1} \ll \sum_{1 \leqq d_{1}, d_{2} \leqq Q} Q^{2}\left(\|D G\|_{\infty} \frac{|\mathbf{I}|}{\left[d_{1}, d_{2}\right]}+\frac{2}{\left[d_{1}, d_{2}\right]^{2}}\right)\right) .
$$

Since 


$$
\begin{aligned}
\sum_{1 \leqq d_{1}, d_{2} \leqq Q} \frac{1}{\left[d_{1}, d_{2}\right]} & =\sum_{1 \leqq \delta \leqq Q} \sum_{\substack{1 \leqq d_{1}, d_{2} \leqq Q \\
\left(d_{1}, d_{2}\right)=\delta}} \frac{1}{\left[d_{1}, d_{2}\right]}=\sum_{1 \leqq \delta \leqq Q} \sum_{\substack{1 \leqq q_{1}, q_{2} \leqq Q,\left(q_{1}, q_{2}\right)=1}} \frac{1}{\delta q_{1} q_{2}} \\
& \ll(\log Q)^{3},
\end{aligned}
$$

and

$$
\sum_{1 \leqq d_{1}, d_{2} \leqq Q} \frac{1}{\left[d_{1}, d_{2}\right]^{2}} \leqq \sum_{1 \leqq \delta \leqq Q} \sum_{\substack{1 \leqq q_{1}, q_{2} \leqq Q \\\left(q_{1}, q_{2}\right)=1}} \frac{1}{\delta^{2} q_{1}^{2} q_{2}^{2}} \leqq\left(\sum_{1 \leqq \delta \leqq Q} \frac{1}{\delta^{2}}\right)^{3}=O(1)
$$

it follows that

$$
E_{G, 1} \ll Q^{2}(\log Q)^{3}\|D G\|_{\infty}|\mathbf{I}|
$$

Moreover we observe that

$$
\begin{aligned}
\sum_{d \leqq Q} d M\left(\frac{Q}{d}\right) & =\sum_{d \leqq Q} d \sum_{r \leqq Q / d} \mu(r)=\sum_{r \leqq Q} \mu(r) \sum_{d \leqq Q / r} d \\
& =\sum_{r \leqq Q} \mu(r)\left(\frac{Q}{r}+O(1)\right)^{2} \\
& =\frac{Q^{2}}{2} \sum_{r \leqq Q} \frac{\mu(r)}{r^{2}}+O(Q \log Q),
\end{aligned}
$$

and therefore

$$
\begin{aligned}
\sum_{d \leqq Q} d M\left(\frac{Q}{d}\right) & \left.=\frac{Q^{2}}{2} \quad \frac{6}{\pi^{2}}+O\left(\frac{1}{Q}\right)\right)+O(Q \log Q) \\
& =\frac{3 Q^{2}}{\pi^{2}}+O(Q \log Q),
\end{aligned}
$$

which finally gives

$$
\left(\sum_{1 \leqq d \leqq Q} d M\left(\frac{Q}{d}\right)\right)^{2}=\frac{9 Q^{4}}{\pi^{4}}+O\left(Q^{3} \log Q\right) .
$$

Combining all these estimates completes the proof of Lemma 2.

Proof of Lemma 1. We will approximate the characteristic function $\chi_{\mathbf{I}}$ of $\mathbf{I}$ by a $C^{1}$ function. To this end, consider the function $f(x)=3 x^{2}-2 x^{3}$ for $x \in[0,1]$. First note the following properties:

- $f^{\prime}(x)=6 x(1-x) \geqq 0$ and $\left|f^{\prime}(x)\right| \leqq 3 / 2$ for $x \in[0,1]$;

- $f^{\prime}(0)=f^{\prime}(1)=0, f(0)=0, f(1)=1$;

- $\int_{0}^{1} f(x) \mathrm{d} x=1 / 2$. 
For real numbers $a<b<c<d$, we define the function $g_{a, b, c, d}: \mathbb{R} \rightarrow[0,1]$ by

$$
g_{a, b, c, d}(t)= \begin{cases}0, & t \leqq a \\ f\left(\frac{t-a}{b-a}\right), & a<t \leqq b \\ 1, & b<t \leqq c \\ f\left(1-\frac{t-c}{d-c}\right), & c<t \leqq d \\ 0, & d<t .\end{cases}
$$

It is easy to see that $g_{a, b, c, d} \in C^{1}(\mathbb{R})$ with $\operatorname{Supp}\left(g_{a, b, c, d}\right) \subset[a, d]$, and

$$
\begin{aligned}
\left\|D g_{a, b, c, d}\right\|_{\infty} \leqq \frac{3}{2} \max \left(\frac{1}{b-a}, \frac{1}{d-c}\right), \\
\int_{\mathbb{R}} g_{a, b, c, d}(x) \mathrm{d} x=c-b+\frac{b-a}{2}+\frac{d-c}{2} .
\end{aligned}
$$

Now let $G=\chi_{[a, b]}$, the characteristic function of interval $\mathbf{I}=[a, b] \subset[0,1]$. Putting $a_{1}=a-\varepsilon, a_{2}=a+\varepsilon, b_{1}=b+\varepsilon, b_{2}=b-\varepsilon$ and $G_{1}=g_{a_{1}, a, b, b_{1}}, G_{2}=g_{a, a_{2}, b_{2}, b}$, we may denote by

$$
f(y)=\sum_{n \in \mathbb{Z}} G(y+n), \quad f_{1}(y)=\sum_{n \in \mathbb{Z}} G_{1}(y+n), \quad f_{2}(y)=\sum_{n \in \mathbb{Z}} G_{2}(y+n),
$$

to obtain that

$$
S_{Q, G}=\sum_{\gamma, \gamma^{\prime} \in \mathscr{F}_{Q}} f\left(\gamma+\gamma^{\prime}\right), \quad S_{Q, G_{1}}=\sum_{\gamma, \gamma^{\prime} \in \mathscr{F}_{Q}} f_{1}\left(\gamma+\gamma^{\prime}\right), \quad S_{Q, G_{2}}=\sum_{\gamma, \gamma^{\prime} \in \mathscr{F}_{Q}} f_{2}\left(\gamma+\gamma^{\prime}\right) .
$$

Since $G_{2} \leqq G=\chi_{[a, b]} \leqq G_{1}$, we have

$$
S_{Q, G_{2}} \leqq S_{Q, G} \leqq S_{Q, G_{1}}
$$

Noticing that

$$
\int_{\mathbb{R}} G_{1}(x) \mathrm{d} x=b-a+\varepsilon=|\mathbf{I}|+\varepsilon, \quad \int_{\mathbb{R}} G_{2}(x) \mathrm{d} x=b-a-\varepsilon=|\mathbf{I}|-\varepsilon,
$$

and

$$
\left\|D G_{1}\right\|_{\infty} \leqq \frac{3}{\varepsilon}, \quad\left\|D G_{2}\right\|_{\infty} \leqq \frac{3}{\varepsilon}
$$

we may use Lemma 2 to obtain

$$
\begin{aligned}
& S_{Q, G_{1}}=(|\mathbf{I}|+\varepsilon) \frac{9}{\pi^{4}} Q^{4}+E_{Q, G_{1}}, \\
& S_{Q, G_{2}}=(|\mathbf{I}|-\varepsilon) \frac{9}{\pi^{4}} Q^{4}+E_{Q, G_{2}},
\end{aligned}
$$


where

$$
E_{Q, G_{1}} \ll Q^{2}(\log Q)^{3} \frac{3}{\varepsilon}(|\mathbf{I}|+2 \varepsilon)+Q^{3} \log Q(|\mathbf{I}|+\varepsilon)
$$

and

$$
E_{Q, G_{2}} \ll Q^{2}(\log Q)^{3} \frac{3}{\varepsilon}|\mathbf{I}|+Q^{3} \log Q(|\mathbf{I}|-\varepsilon) .
$$

Choosing

$$
\varepsilon=\frac{(\log Q)^{3 / 2}}{Q}
$$

we have

$$
S_{Q, G_{1}}=S_{Q, G_{2}}=\frac{9|\mathbf{I}|}{\pi^{4}} Q^{4}+E
$$

where

$$
E \ll Q^{3}(\log Q)^{3 / 2}
$$

Therefore

$$
\# \mathcal{G}_{\mathbf{I}}(Q)=S_{Q, G}=\frac{9|\mathbf{I}|}{\pi^{4}} Q^{4}+O\left(Q^{3}(\log Q)^{3 / 2}\right),
$$

which completes the proof of Lemma 1.

\section{Pair correlation of sums of Farey fractions}

For each positive integer $Q$, let $\mathscr{F}_{Q}=\left\{\gamma_{1}, \ldots, \gamma_{N(Q)}\right\}$ denote the Farey sequence of order $Q$ with $1 / Q=\gamma_{1}<\gamma_{2}<\cdots<\gamma_{N(Q)}=1$. Let I be a subinterval of $[0,1]$. Denote by $x_{i j} \equiv \gamma_{i}+\gamma_{j}(\bmod 1)$ and $\mathcal{G}_{Q}:=\left\{x_{i j} \mid 1 \leqq i, j \leqq N(Q)\right\}=\mathscr{F}_{Q}+\mathscr{F}_{Q}(\bmod 1)$, the set of sums of Farey sequences of order $Q$ counted with multiplicity, $\mathcal{G}=\left(\mathcal{G}_{Q}\right)_{Q}$ and $\mathcal{G}_{\mathbf{I}}(Q):=\mathcal{G}_{Q} \cap \mathbf{I}$. Let $\# \mathcal{G}_{\mathbf{I}}(Q)$ be the cardinality of $\mathcal{G}_{\mathbf{I}}(Q)$. It is known from Lemma 1 that

$$
N=\frac{\# \mathcal{G}_{\mathbf{I}}(Q)}{|\mathbf{I}|}=\frac{9 Q^{4}}{\pi^{4}}+O\left(Q^{3}(\log Q)^{3 / 2}\right)
$$

Our goal is to estimate the quantity

$$
S_{Q, \mathbf{I}}(\wedge):=\#\left\{(x, y) \in \mathcal{G}_{\mathbf{I}}(Q) \times \mathcal{G}_{\mathbf{I}}(Q) \mid x \neq y, x-y \in \frac{(0, \wedge)}{N}+\mathbb{Z}\right\}
$$

for any positive real number $\wedge$ as $Q \rightarrow \infty$. In fact we prove a more general result. 
Lemma 3. Given the functions $G, H \in C^{1}(\mathbb{R})$ with $\operatorname{Supp}(G) \subset(0,1)$ and $\operatorname{Supp}(H) \subset(0, \wedge)$ for some $\wedge>0$, define

$$
h(y)=\sum_{n \in \mathbb{Z}} H(N(y+n)), \quad g(y)=\sum_{n \in \mathbb{Z}} G(y+n),
$$

and let

$$
S_{Q, \mathbf{I}, H, G}=\sum_{x, y \in \mathcal{G}_{Q}} h(x-y) g(x) g(y) .
$$

Then we have

$$
S_{Q, \mathbf{I}, H, G}=\frac{9 Q^{4}}{\pi^{4}}\left(\int_{0}^{1} G(z)^{2} \mathrm{~d} z\right) \int_{0}^{\wedge} H(x) g_{2}(x) \mathrm{d} x+E_{Q, \mathbf{I}, H, G}
$$

where for any $x>0$,

$$
g_{2}(x)=\frac{c}{\pi^{2} x^{2}} \sum_{1 \leqq k \leqq \frac{\pi^{4} x}{9}} \psi(k) \log ^{3} \frac{\pi^{4} x}{9 k}
$$

Here

$$
c:=\prod_{p \text { prime }}\left(1-\frac{2}{p(p+1)}\right)\left(1-\frac{3}{p(p+2)}\right)
$$

$\psi$ is the multiplicative function defined in (1) and for any $\eta>0$,

$$
E_{Q, \mathbf{I}, H, G} \ll \mathbf{I}, H, G, \eta Q^{4-\frac{1}{18}+\eta} .
$$
obtain

Note that assuming Lemma 3 with the error term $Q^{4-\frac{1}{18}+\eta}$, for $0<\eta<1 / 18$, we may

$$
\begin{aligned}
\lim _{Q \rightarrow \infty} \frac{S_{Q, \mathbf{I}, H, G}}{\# \mathcal{G}_{\mathbf{I}}(Q)} & =\lim _{Q \rightarrow \infty} \frac{S_{Q, \mathbf{I}, H, G}}{\frac{9|\mathbf{I}|}{\pi^{4}} Q^{4}} \\
& =\frac{\int_{0}^{1} G(z)^{2} \mathrm{~d} z}{|\mathbf{I}|} \int_{0} H(x) g_{2}(x) \mathrm{d} x .
\end{aligned}
$$

Let the smooth function $G$ approach $\chi_{\mathbf{I}}$, the characteristic function of the interval $\mathbf{I}$, so that

$$
\frac{\int_{0}^{1} G(z)^{2} \mathrm{~d} z}{|\mathbf{I}|} \rightarrow 1
$$

Also let the smooth function $H$ approach $\chi_{(0, \wedge)}$, the characteristic function of the interval $(0, \wedge)$. By a standard approximation argument, we see that the pair correlation function of 
$\mathcal{G}$ along the subinterval I of $[0,1]$ exists and is independent of the location and length of the subinterval. This completes the proof of Theorem 1 .

Proof of Lemma 3. The proof of Lemma 3 will require several steps. Throughout the proof, all constants implied by the big " $O$ " or " $\ll$ " notation may depend on the functions $H$ and $G$.

3.1. Fourier series expansion and Poisson summation formula. If the Fourier series expansion of the functions $h$ and $g$ are given by

$$
h(y)=\sum_{n \in \mathbb{Z}} c_{n} e(n y)
$$

and

$$
g(y)=\sum_{n \in \mathbb{Z}} a_{n} e(n y)
$$

for $y \in \mathbb{R}$, then it follows that

$$
\begin{aligned}
S_{Q, \mathbf{I}, H, G}= & \sum_{\gamma_{1}, \gamma_{2}, \gamma_{1}^{\prime}, \gamma_{2}^{\prime} \in \mathscr{F}_{Q}} \sum_{m} c_{m} e\left(m\left(\gamma_{1}+\gamma_{2}\right)-m\left(\gamma_{1}^{\prime}+\gamma_{2}^{\prime}\right)\right) \\
& \times \sum_{n} a_{n} e\left(n\left(\gamma_{1}+\gamma_{2}\right)\right) \sum_{r} a_{r} e\left(r\left(\gamma_{1}^{\prime}+\gamma_{2}^{\prime}\right)\right) \\
= & \sum_{m, n, r} c_{m} a_{n} a_{r} \sum_{\gamma_{1} \in \mathscr{F}_{Q}} e\left((m+n) \gamma_{1}\right) \sum_{\gamma_{2} \in \mathscr{F}_{Q}} e\left((m+n) \gamma_{2}\right) \\
& \times \sum_{\gamma_{1}^{\prime} \in \mathscr{F}_{Q}} e\left((r-m) \gamma_{1}^{\prime}\right) \sum_{\gamma_{2}^{\prime} \in \mathscr{F}_{Q}} e\left((r-m) \gamma_{2}^{\prime}\right) \\
= & \sum_{m, n, r} c_{m} a_{n} a_{r}\left(\sum_{\gamma \in \mathscr{F}_{Q}} e((m+n) \gamma)\right)^{2}\left(\sum_{\gamma \in \mathscr{F}_{Q}} e((r-m) \gamma)\right)^{2} .
\end{aligned}
$$

Therefore

$$
\left.S_{Q, \mathbf{I}, H, G}=\sum_{m, n, r} c_{m} a_{n} a_{r} \sum_{\substack{1 \leqq d \leqq Q, \bar{d} \mid m+n}} d M\left(\frac{Q}{d}\right)\right)^{2}\left(\sum_{\substack{1 \leqq d \leqq Q, d \mid r-m}} d M\left(\frac{Q}{d}\right)\right)^{2}
$$

Changing the summation indices using $m+n=m^{\prime}, r-m=n^{\prime}, m=r^{\prime}$, we have $m=r^{\prime}$, $n=m^{\prime}-r^{\prime}, r=n^{\prime}+r^{\prime}$. Consequently in terms of $m^{\prime}, n^{\prime}, r^{\prime}$, we have

$$
\begin{aligned}
S_{Q, \mathbf{I}, H, G} & \left.=\sum_{m^{\prime}, n^{\prime}, r^{\prime}} c_{r^{\prime}} a_{m^{\prime}-r^{\prime}} a_{n^{\prime}+r^{\prime}} \sum_{\substack{1 \leqq d \leqq Q, d \mid m^{\prime}}} d M\left(\frac{Q}{d}\right)\right)^{2}\left(\sum_{\substack{1 \leqq d \leqq Q, d \mid n^{\prime}}} d M\left(\frac{Q}{d}\right)\right)^{2} \\
& =\sum_{1 \leqq d_{1}, d_{2}, d_{3}, d_{4} \leqq Q} d_{1} \cdots d_{4} M\left(\frac{Q}{d_{1}}\right) \cdots M\left(\frac{Q}{d_{4}}\right) \sum_{\substack{r, m, n \in \mathbb{Z}, d_{1}\left|m, d_{2}\right| m, d_{3}\left|n, d_{4}\right| n}} c_{r} a_{m-r} a_{n+r} .
\end{aligned}
$$

Using an argument similar to that from [8] with Poisson summation formula, the inner sum on $r, m, n$ is given as 


$$
\begin{aligned}
& \sum_{r \in \mathbb{Z}} c_{r} \sum_{m \in \mathbb{Z}} a_{\left[d_{1}, d_{2}\right] m-r} \sum_{n \in \mathbb{Z}} a_{\left[d_{3}, d_{4}\right] n+r} \\
& =\sum_{m, n} \frac{G\left(\frac{m}{\left[d_{1}, d_{2}\right]}\right) G\left(\frac{n}{\left[d_{3}, d_{4}\right]}\right)}{\left[d_{1}, d_{2}\right]\left[d_{3}, d_{4}\right]} \sum_{r} c_{r} e\left(\left(\frac{m}{\left[d_{1}, d_{2}\right]}-\frac{n}{\left[d_{3}, d_{4}\right]}\right) r\right) \\
& \left.=\sum_{m, n} \frac{G\left(\frac{m}{\left[d_{1}, d_{2}\right]}\right) G\left(\frac{n}{\left[d_{3}, d_{4}\right]}\right)}{\left[d_{1}, d_{2}\right]\left[d_{3}, d_{4}\right]} \sum_{r} H \quad N\left(r+\frac{m}{\left[d_{1}, d_{2}\right]}-\frac{n}{\left[d_{3}, d_{4}\right]}\right)\right) .
\end{aligned}
$$

3.2. Further reductions. We need several reductions to convert the expression of $S_{Q, \mathbf{I}, H, G}$ to a manageable form.

3.2.1. First reduction. First of all, note that since

$$
\operatorname{Supp}(G) \subset(0,1), \quad \operatorname{Supp}(H) \subset(0, \wedge),
$$

$\left[d_{1}, d_{2}\right],\left[d_{3}, d_{4}\right] \leqq Q^{2}$ and $N \sim \frac{9}{\pi^{4}} Q^{4}$, if $r \neq 0$, then as $Q$ is sufficiently large, we have

$$
H\left(N\left(r+\frac{m}{\left[d_{1}, d_{2}\right]}-\frac{n}{\left[d_{3}, d_{4}\right]}\right)\right)=0
$$

Hence we may assume that $r=0$.

3.2.2. Second reduction. For positive integers $d_{1}, d_{2}, d_{3}, d_{4}$, let $\tilde{u}=\left(\left[d_{1}, d_{2}\right],\left[d_{3}, d_{4}\right]\right)$ and

$$
e_{1}=\frac{\left[d_{1}, d_{2}\right]}{\tilde{u}}, \quad e_{2}=\frac{\left[d_{3}, d_{4}\right]}{\tilde{u}}
$$

Since $\left(e_{1}, e_{2}\right)=1$, there is a unique integer $\tilde{a}_{2}$ such that $0<\tilde{a}_{2}<e_{1}, \tilde{a}_{2} e_{2} \equiv 1\left(\bmod e_{1}\right)$. Choose $\tilde{a}_{1}=\left(1-\tilde{a}_{2} e_{2}\right) / e_{1}$, so that $\tilde{a}_{1} e_{1}+\tilde{a}_{2} e_{2}=1$. Changing the summation indices with $m^{\prime}=e_{2} m-e_{1} n, n^{\prime}=\tilde{a}_{1} m+\tilde{a}_{2} n$, we have $m=\tilde{a}_{2} m^{\prime}+e_{1} n^{\prime}, n=-\tilde{a}_{1} m^{\prime}+e_{2} n^{\prime}$, and hence

$$
\begin{aligned}
\left.\sum_{m, n \in \mathbb{Z}} G\left(\frac{m}{\left[d_{1}, d_{2}\right]}\right) G\left(\frac{n}{\left[d_{3}, d_{4}\right]}\right) H N\left(\frac{m}{\left[d_{1}, d_{2}\right]}-\frac{n}{\left[d_{3}, d_{4}\right]}\right)\right) \\
=\sum_{m^{\prime}, n^{\prime} \in \mathbb{Z}} G\left(\frac{\tilde{a}_{2} m^{\prime}}{\left[d_{1}, d_{2}\right]}+\frac{n^{\prime}}{\tilde{u}}\right) G\left(\frac{-\tilde{a}_{1} m^{\prime}}{\left[d_{3}, d_{4}\right]}+\frac{n^{\prime}}{\tilde{u}}\right) H\left(\frac{N m^{\prime}}{\left[d_{1}, d_{2}, d_{3}, d_{4}\right]}\right) .
\end{aligned}
$$

Using

$$
M\left(\frac{Q}{d}\right)=\sum_{r \leqq Q / d} \mu(r)
$$

and changing the order of summation we rewrite $S_{Q, \mathbf{I}, H, G}$ as 


$$
\begin{aligned}
S_{Q, \mathbf{I}, H, G}= & \sum_{1 \leqq r_{1}, r_{2}, r_{3}, r_{4} \leqq Q} \mu\left(r_{1}\right) \mu\left(r_{2}\right) \mu\left(r_{3}\right) \mu\left(r_{4}\right) \sum_{\substack{d_{1} \leqq Q / r_{1} \\
\vdots \\
d_{4} \leqq Q / r_{4}}} \frac{d_{1} d_{2} d_{3} d_{4}}{\left[d_{1}, d_{2}\right]\left[d_{3}, d_{4}\right]} \\
& \times \sum_{m, n \in \mathbb{Z}} G\left(\frac{\tilde{a}_{2} m}{\left[d_{1}, d_{2}\right]}+\frac{n}{\tilde{u}}\right) G\left(\frac{-\tilde{a}_{1} m}{\left[d_{3}, d_{4}\right]}+\frac{n}{\tilde{u}}\right) H\left(\frac{N m}{\left[d_{1}, d_{2}, d_{3}, d_{4}\right]}\right) .
\end{aligned}
$$

3.2.3. Third reduction. For positive integers $d_{1}, d_{2}, d_{3}, d_{4}$, denote

$$
\delta=\frac{d_{1} d_{2} d_{3} d_{4}}{\left[d_{1}, d_{2}, d_{3}, d_{4}\right]} .
$$

If $Q$ is sufficiently large, then

$$
\frac{9 Q^{4}}{\pi^{4}}(1-\varepsilon)<N<\frac{9 Q^{4}}{\pi^{4}}(1+\varepsilon)
$$

for any $0<\varepsilon<1$. Since $\operatorname{Supp}(H) \subset(0, \wedge)$, to have a non-zero contribution from $H$, we need

$$
\begin{aligned}
0<m r_{1} r_{2} r_{3} r_{4} \frac{9}{\pi^{4}}(1-\varepsilon) \cdot \delta & <m r_{1} r_{2} r_{3} r_{4} \frac{N}{Q^{4}} \cdot \delta \\
& \leqq m r_{1} r_{2} r_{3} r_{4} \frac{N}{r_{1} r_{2} r_{3} r_{4} d_{1} d_{2} d_{3} d_{4}} \cdot \delta \\
& =\frac{N m}{\left[d_{1}, d_{2}, d_{3}, d_{4}\right]}<\wedge,
\end{aligned}
$$

which reduces to the condition

$$
m r_{1} r_{2} r_{3} r_{4} \delta<\frac{\pi^{4} \wedge}{9(1-\varepsilon)}
$$

Denoting

$$
C_{\wedge}=\frac{\pi^{4} \wedge}{9}
$$

and choosing $\varepsilon$ sufficiently small, we have

$$
1 \leqq m r_{1} r_{2} r_{3} r_{4} \delta \leqq C_{\wedge}
$$

3.2.4. Fourth reduction. Fix $m, r_{1}, r_{2}, r_{3}, r_{4}$ and $\delta=\frac{d_{1} d_{2} d_{3} d_{4}}{\left[d_{1}, d_{2}, d_{3}, d_{4}\right]}$ bounded by $C_{\wedge}$. Since $\tilde{u} \mid \delta$, and $\operatorname{Supp}(G) \subset[0,1]$, to have a non-zero contribution from $G$, we need

$$
0<\frac{\tilde{a}_{2} m}{\left[d_{1}, d_{2}\right]}+\frac{n}{\tilde{u}}<1
$$


There are only finitely many integers $n$ satisfying this inequality. Denote by $\mathcal{A}$ the finite set consisting of all possible values of such $n$. Changing the order of summation we obtain that

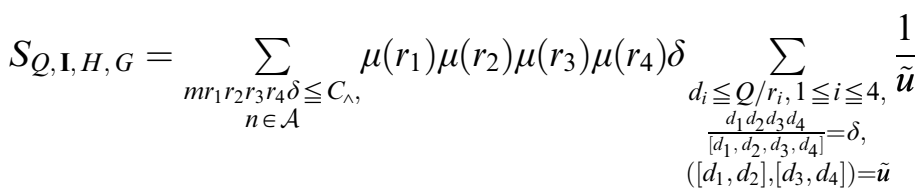

$$
\begin{aligned}
& \times G\left(\frac{\tilde{a}_{2} m}{\left[d_{1}, d_{2}\right]}+\frac{n}{\tilde{u}}\right) G\left(\frac{-\tilde{a}_{1} m}{\left[d_{1}, d_{2}\right]}+\frac{n}{\tilde{u}}\right) H\left(\frac{N m \delta}{d_{1} d_{2} d_{3} d_{4}}\right) .
\end{aligned}
$$

3.2.5. Fifth reduction. Since $\tilde{a}_{1} e_{1}+\tilde{a}_{2} e_{2}=1$, we have

$$
\frac{\tilde{a}_{1}}{\left[d_{3}, d_{4}\right]}+\frac{\tilde{a}_{2}}{\left[d_{1}, d_{2}\right]}=\frac{\tilde{u}}{\left[d_{1}, d_{2}\right] \cdot\left[d_{3}, d_{4}\right]},
$$

and it follows that

$$
\begin{aligned}
& \left|\frac{\tilde{a}_{2} m}{\left[d_{1}, d_{2}\right]}+\frac{n}{\tilde{u}}-\left(\frac{-\tilde{a}_{1} m}{\left[d_{1}, d_{2}\right]}+\frac{n}{\tilde{u}}\right)\right|=\left|\frac{\tilde{a}_{2} m}{\left[d_{1}, d_{2}\right]}-\frac{-\tilde{a}_{1} m}{\left[d_{1}, d_{2}\right]}\right| \\
& =\frac{m u}{\left[d_{1}, d_{2}\right] \cdot\left[d_{3}, d_{4}\right]}=\frac{m}{\left[d_{1}, d_{2}, d_{3}, d_{4}\right]}=\frac{m \delta}{d_{1} d_{2} d_{3} d_{4}} \leqq \frac{C_{\curlywedge}}{d_{1} d_{2} d_{3} d_{4}}
\end{aligned}
$$

and

$$
G\left(\frac{-\tilde{a}_{1} m}{\left[d_{1}, d_{2}\right]}+\frac{n}{\tilde{u}}\right)=G\left(\frac{\tilde{a}_{2} m}{\left[d_{1}, d_{2}\right]}+\frac{n}{\tilde{u}}\right)+O\left(\frac{1}{d_{1} d_{2} d_{3} d_{4}}\right)
$$

As a result of this reduction, we get

$$
S_{Q, \mathbf{I}, H, G}=\sum_{\substack{m r_{1} r_{2} r_{3} r_{4} \delta \leqq C_{\curlywedge}, n \in \mathcal{A}}} \mu\left(r_{1}\right) \mu\left(r_{2}\right) \mu\left(r_{3}\right) \mu\left(r_{4}\right) \delta \cdot \sum+E_{0},
$$

where the inner sum is given by

$$
\sum=\sum_{\substack{d_{i} \leqq Q / r_{i}, 1 \leqq i \leqq 4 \\ \text { a } \\\left[d_{1} d_{2} d_{3} d_{4} \\\left[d_{1}, d_{2}, d_{3}, d_{4}=\delta,\left(d_{1}, d_{2}\right],\left[d_{3}, d_{4}\right]\right)=\tilde{u}\right.}} \frac{1}{\tilde{u}} G\left(\frac{\tilde{a}_{2} m}{\left[d_{1}, d_{2}\right]}+\frac{n}{\tilde{u}}\right)^{2} H\left(\frac{N m \delta}{d_{1} d_{2} d_{3} d_{4}}\right)
$$

Moreover the error term $E_{0}$ can be estimated as

$$
E_{0} \ll \sum_{1 \leqq d_{1}, d_{2}, d_{3}, d_{4} \leqq Q} \frac{1}{\tilde{u} \cdot d_{1} d_{2} d_{3} d_{4}} \ll(\log Q)^{4} \ll_{\eta} Q^{\eta}
$$

for any $\eta>0$.

3.2.6. Sixth reduction. Fix integers $m, r_{1}, r_{2}, r_{3}, r_{4}, \delta, n$ and $u$. Define

$$
P_{\delta}=\{a \in \mathbb{N} \mid \text { for any prime } p, p|a \Rightarrow p| \delta\} .
$$


For positive integers $d_{1}, d_{2}, d_{3}, \quad d_{4}, d_{i} \leqq Q / r_{i}, 1 \leqq i \leqq 4$ with $\frac{d_{1} d_{2} d_{3} d_{4}}{\left[d_{1}, d_{2}, d_{3}, d_{4}\right]}$ and $\left(\left[d_{1}, d_{2}\right],\left[d_{3}, d_{4}\right]\right)=\tilde{u}$, factoring $d_{1}, d_{2}, d_{3}, d_{4}$ as $d_{1}=a_{1} q_{1}, d_{2}=a_{2} q_{2}, d_{3}=a_{3} q_{3}, d_{4}=a_{4} q_{4}$ with $a_{i} \in P_{\delta}$ and $\left(q_{i}, \delta\right)=1$ for $1 \leqq i \leqq 4$ together with $\left[d_{1}, d_{2}\right]=\left[a_{1}, a_{2}\right] q_{1} q_{2}$, $\tilde{u}=\left(\left[d_{1}, d_{2}\right],\left[d_{3}, d_{4}\right]\right)=\left(\left[a_{1}, a_{2}\right],\left[a_{3}, a_{4}\right]\right)$, and $\frac{d_{1} d_{2} d_{3} d_{4}}{\left[d_{1}, d_{2}, d_{3}, d_{4}\right]}=\delta$ implies that

$$
\frac{a_{1} a_{2} a_{3} a_{4}}{\left[a_{1}, a_{2}, a_{3}, a_{4}\right]}=\delta, \quad \frac{q_{1} q_{2} q_{3} q_{4}}{\left[q_{1}, q_{2}, q_{3}, q_{4}\right]}=1
$$

Here $\left(q_{i}, q_{j}\right)=1$ for $i \neq j$. Using these observations we can rewrite $\sum$ in (5) as

$$
\sum=\sum_{\substack{a_{i} \leqq Q / r_{i}, 1 \leqq i \leqq 4 \\ a_{1}, a_{2}, a_{3}, a_{4} \in \bar{P}_{\delta} \\ \frac{a_{1} a_{2} a_{3} a_{4}}{\left[a_{1}, a_{2}, a_{3}, a_{4}\right]}=\delta \\\left(\left[a_{1}, a_{2}\right],\left[a_{3}, a_{4}\right]\right)=\tilde{u}}} \frac{1}{\tilde{u}} \cdot \sum_{1},
$$

where the inner sum in (8) is given by

(9) $\quad \sum_{1}=\sum_{\substack{q_{i} \leqq Q / a_{i} r_{i}, 1 \leqq i \leqq 4 \\\left(q_{i}, q_{j}=1, i \neq j,\left(q_{i}, \delta\right)=1\right.}} G\left(\frac{1}{\tilde{u}}\left(\frac{\tilde{a}_{2} m}{\frac{\left[a_{1}, a_{2}\right] q_{1} q_{2}}{\tilde{u}}}+n\right)\right)^{2} H\left(\frac{N m \delta}{a_{1} a_{2} a_{3} a_{4} q_{1} q_{2} q_{3} q_{4}}\right)$.

3.2.7. Seventh reduction. Next fix positive integers $a_{1}, a_{2}, a_{3}, a_{4} \in P_{\delta}$. Let

$$
a=\frac{\left[a_{1}, a_{2}\right]}{\tilde{u}}, \quad b=\frac{\left[a_{3}, a_{4}\right]}{\tilde{u}}, \quad \text { so that }(a, b)=1 \text { and } a|\delta, b| \delta, \tilde{u} \mid \delta
$$

Define the functions

$$
f(x)=G\left(\frac{1}{\tilde{u}} \cdot(m x+n)\right)^{2}, \quad h(x, y, z, w)=H\left(\frac{N \lambda}{x y z w}\right),
$$

where

$$
\lambda=\frac{m \delta}{a_{1} a_{2} a_{3} a_{4}} \neq 0
$$

We have

$$
\begin{aligned}
& e_{1}=\frac{\left[d_{1}, d_{2}\right]}{\tilde{u}}=\frac{\left[a_{1}, a_{2}\right] q_{1} q_{2}}{\tilde{u}}=a q_{1} q_{2} \leqq Q^{2}, \\
& e_{2}=\frac{\left[d_{3}, d_{4}\right]}{\tilde{u}}=\frac{\left[a_{3}, a_{4}\right] q_{3} q_{4}}{\tilde{u}}=b q_{3} q_{4} \leqq Q^{2},
\end{aligned}
$$

and 


$$
0<\tilde{a}_{2}<a q_{1} q_{2}, \quad \tilde{a}_{2}\left(b q_{3} q_{4}\right) \equiv 1\left(\bmod a q_{1} q_{2}\right)
$$

Denoting

$$
\delta_{i}=a_{i} r_{i} \geqq 1, \quad 1 \leqq i \leqq 4,
$$

we can rewrite $(9)$ in the form

$$
\sum_{1}=\sum_{\substack{q_{i} \leqq Q / \delta_{i}, 1 \leqq i \leqq 4,\left(q_{i}, q_{j}\right)=1, i \neq j,\left(q_{i}, \delta\right)=1}} f\left(\frac{\tilde{a}_{2}}{a q_{1} q_{2}}\right) h\left(q_{1}, q_{2}, q_{3}, q_{4}\right) .
$$

3.3. Further estimations. We will need some further estimations in several stages.

3.3.1. First step. We know that

$$
\|f\|_{\infty}=O(1), \quad\|D f\|_{\infty}=O(1)
$$

Choosing $0<\varepsilon<1 / 2$, one has

$$
\frac{9 Q^{4}}{2 \pi^{4}}<\frac{9 Q^{4}}{\pi^{4}}(1-\varepsilon)<N<\frac{9 Q^{4}}{\pi^{4}}(1+\varepsilon)<\frac{27 Q^{4}}{2 \pi^{4}}
$$

for $Q$ sufficiently large. Since $\operatorname{Supp}(H) \subset(0, \wedge)$, and $h(x, y, z, w) \neq 0$ for $0<x \leqq Q / \delta_{1}$, $0<y \leqq Q / \delta_{2}, 0<z \leqq Q / \delta_{3}$ and $0<w \leqq Q / \delta_{4}$, we must have that $0<\frac{N \lambda}{x y z w}<\wedge$. This
implies that

$$
\frac{Q}{\delta_{1}} \geqq x>\frac{N \lambda}{\wedge y z w} \geqq \frac{\frac{9 Q^{4} m \delta}{2 \pi^{4} a_{1} a_{2} a_{3} a_{4}}}{\frac{Q^{3}}{a_{2} r_{2} a_{3} r_{3} a_{4} r_{4}} \wedge}=\frac{9 m \delta r_{1} r_{2} r_{3} r_{4}}{2 \pi^{4} \wedge} \frac{Q}{\delta_{1}}
$$

Similar lower bounds can be obtained for $y, z, w$ too. Denoting

$$
c_{\wedge}=\frac{9 m \delta r_{1} r_{2} r_{3} r_{4}}{2 \pi^{4} \wedge}
$$

we have

$$
h(x, y, z, w) \neq 0 \Rightarrow \begin{aligned}
& c_{\wedge} \cdot Q / \delta_{1} \leqq x \leqq Q / \delta_{1}, \\
& c_{\wedge} \cdot Q / \delta_{2} \leqq y \leqq Q / \delta_{2}, \\
& \\
& c_{\wedge} \cdot Q / \delta_{3} \leqq z \leqq Q / \delta_{3}, \\
& \\
& c_{\wedge} \cdot Q / \delta_{4} \leqq w \leqq Q / \delta_{4} .
\end{aligned}
$$

Next for the function $h$, using

$$
\|h\|_{\infty}=O(1),
$$


and

$$
\left|\frac{\partial h}{\partial x}(x, y, z, w)\right|=\left|H^{\prime}\left(\frac{N \lambda}{x y z w}\right)\right| \cdot \frac{N \lambda}{x y z w} \cdot \frac{1}{x},
$$

from (18), we obtain that

$$
\left|\frac{\partial h}{\partial x}(x, y, z, w)\right| \leqq\|D H\|_{\infty} \cdot \wedge \cdot \frac{\delta_{1}}{c_{\wedge} Q} \ll \frac{\delta_{1}}{Q}
$$

Similarly,

$$
\begin{aligned}
\left|\frac{\partial h}{\partial y}(x, y, z, w)\right| & \ll \frac{\delta_{2}}{Q}, \\
\left|\frac{\partial h}{\partial z}(x, y, z, w)\right| & \ll \frac{\delta_{3}}{Q}, \\
\left|\frac{\partial h}{\partial w}(x, y, z, w)\right| & \ll \frac{\delta_{4}}{Q} .
\end{aligned}
$$

3.3.2. Second step. We rewrite (15) in the form

$$
\sum_{1}=\sum_{\substack{q_{1} \leqq Q / \delta_{1}, q_{2} \leqq Q / \delta_{2},\left(q_{1}, q_{2} \delta\right)=1,\left(q_{2}, \delta\right)=1}} \sum_{\substack{q_{3} \leqq Q / \delta_{3}, q_{4} \leqq Q / \delta_{4},\left(q_{3}, q_{1} q_{2} \delta \delta=1,\left(q_{4}, q_{1} q_{2} q_{3} \delta\right)=1\right.}} f\left(\frac{\tilde{a}_{2}}{a q_{1} q_{2}}\right) h\left(q_{1}, q_{2}, q_{3}, q_{4}\right) .
$$

Fixing $q_{1}, q_{2}$, we may denote the inner sum in (24) as

$$
\sum_{2}=\sum_{\substack{q_{3} \leqq Q / \delta_{3}, q_{4} \leqq Q / \delta_{4},\left(q_{3}, q_{1} q_{2} \delta=1,\left(q_{4}, q_{1} q_{2} q_{3} \delta\right)=1\right.}} f\left(\frac{\tilde{a}_{2}}{a q_{1} q_{2}}\right) h\left(q_{1}, q_{2}, q_{3}, q_{4}\right) .
$$

Let $K_{3}, K_{4}$ be large positive integers to be chosen later and $T_{3}, T_{4}$ be real numbers such that

$$
T_{3} K_{3}=\frac{Q}{\delta_{3}}, \quad T_{4} K_{4}=\frac{Q}{\delta_{4}}
$$

Therefore (25) becomes

$$
\sum_{2}=\sum_{\substack{1 \leqq k_{3} \leqq K_{3},\left(k_{3}-1\right) T_{3}<q_{3} \leqq k_{3} T_{3}, 1 \leqq k_{4} \leqq K_{4}\left(k_{4}-1\right) T_{4}<q_{4} \leqq k_{4} T_{4},\left(q_{3}, q_{1} q_{2} \delta\right)=1,\left(q_{4}, q_{1} q_{2} q_{3} \delta\right)=1}} f\left(\frac{\tilde{a}_{2}}{a q_{1} q_{2}}\right) h\left(q_{1}, q_{2}, q_{3}, q_{4}\right) .
$$

Since $\left(k_{3}-1\right) T_{3}<q_{3} \leqq k_{3} T_{3}=\frac{Q}{\delta_{3}} \frac{k_{3}}{K_{3}},\left(k_{4}-1\right) T_{4}<q_{4} \leqq k_{4} T_{4}=\frac{Q}{\delta_{4}} \frac{k_{4}}{K_{4}}$, using (22) and
(23) we have 


$$
\left|h\left(q_{1}, q_{2}, q_{3}, q_{4}\right)-h\left(q_{1}, q_{2}, \frac{Q}{\delta_{3}} \frac{k_{3}}{K_{3}}, \frac{Q}{\delta_{4}} \frac{k_{4}}{K_{4}}\right)\right| \ll \frac{\left(\delta_{3}+\delta_{4}\right)\left(T_{3}+T_{4}\right)}{Q} .
$$

Inserting this into (26), we deduce that

$$
\sum_{2}=\sum_{\substack{1 \leqq k_{3} \leqq K_{3}, 1 \leqq k_{4} \leqq K_{4}}} h\left(q_{1}, q_{2}, \frac{Q}{\delta_{3}} \frac{k_{3}}{K_{3}}, \frac{Q}{\delta_{4}} \frac{k_{4}}{K_{4}}\right) \sum_{\begin{array}{c}
\left(k_{3}-1\right) T_{3}<q_{3} \leqq k_{3} T_{3}, \\
\left(k_{4}-1\right) T_{4}<q_{4} \leqq k_{4} T_{4}, \\
\left(q_{3}, q_{1} q_{2} \delta\right)=1, \\
\left(q_{4}, q_{1} q_{2} q_{3} \delta\right)=1
\end{array}} f\left(\frac{\tilde{a}_{2}}{a q_{1} q_{2}}\right)+E_{2}^{\prime},
$$

where the error term $E_{2}^{\prime}$ in $(27)$ can be estimated as

$$
E_{2}^{\prime} \ll \frac{Q}{\delta_{3}} \frac{Q}{\delta_{4}}\left(\frac{\left(\delta_{3}+\delta_{4}\right)\left(T_{3}+T_{4}\right)}{Q}\right) \ll\left(T_{3}+T_{4}\right) Q .
$$

3.3.3. Third step. For fixed $q_{1}, q_{2}, k_{3}, k_{4}$, let $K^{\prime}$ be a large positive integer to be chosen later and let $T^{\prime}$ be a real number such that

$$
T^{\prime} K^{\prime}=a q_{1} q_{2} \leqq Q^{2}
$$

We can now rewrite the inner sum of the main term of $\sum_{2}$ from (27) as

$$
\sum_{3}=\sum_{1 \leqq k^{\prime} \leqq K^{\prime}} \sum_{\begin{array}{c}
\left(k_{3}-1\right) T_{3}<q_{3} \leqq k_{3} T_{3} \\
\left(k_{4}-1\right) T_{4}<q_{4} \leqq k_{4} T_{4} \\
\left(q_{3}, q_{1} q_{2} \delta\right)=1 \\
\left(q_{4}, q_{1} q_{2} q_{3} \delta\right)=1 \\
\left(k^{\prime}-1\right) T^{\prime}<\tilde{a}_{2} \leqq k^{\prime} T^{\prime} \\
\tilde{a}_{2} \cdot b q_{3} q_{4} \equiv 1\left(\bmod a q_{1} q_{2}\right)
\end{array}} f\left(\frac{\tilde{a}_{2}}{a q_{1} q_{2}}\right) .
$$

For $\left(k^{\prime}-1\right) T^{\prime}<\tilde{a}_{2} \leqq k^{\prime} T^{\prime}$, we have

$$
\frac{\left(k^{\prime}-1\right) T^{\prime}}{a q_{1} q_{2}}<\frac{\tilde{a}_{2}}{a q_{1} q_{2}} \leqq \frac{k^{\prime} T^{\prime}}{a q_{1} q_{2}}=\frac{k^{\prime}}{K^{\prime}}
$$

so that

$$
\left|\frac{\tilde{a}_{2}}{a q_{1} q_{2}}-\frac{k^{\prime}}{K^{\prime}}\right| \leqq \frac{T^{\prime}}{a q_{1} q_{2}}=\frac{1}{K^{\prime}}
$$

and

$$
\left|f\left(\frac{\tilde{a}_{2}}{a q_{1} q_{2}}\right)-f\left(\frac{k^{\prime}}{K^{\prime}}\right)\right| \leqq\|D f\|_{\infty} \cdot\left|\frac{\tilde{a}_{2}}{a q_{1} q_{2}}-\frac{k^{\prime}}{K^{\prime}}\right| \ll \frac{1}{K^{\prime}} .
$$

Therefore (29) becomes

$$
\sum_{3}=\sum_{1 \leqq k^{\prime} \leqq K^{\prime}} f\left(\frac{k^{\prime}}{K^{\prime}}\right) \sum_{\begin{array}{c}
\left(k_{3}-1\right) T_{3}<q_{3} \leqq k_{3} T_{3} \\
\left(k_{4}-1\right) T_{4}<q_{4} \leqq k_{4} T_{4} \\
\left(q_{3}, q_{1} q_{2} \delta\right)=1 \\
\left(q_{4}, q_{1} q_{2} q_{3} \delta\right)=1 \\
\left(k^{\prime}-1\right) T^{\prime}<\tilde{a}_{2} \leqq k^{\prime} T^{\prime} \\
\tilde{a}_{2} \cdot b q_{3} q_{4} \equiv 1\left(\bmod a q_{1} q_{2}\right)
\end{array}} 1+E_{3}^{\prime}
$$


where the error term $E_{3}^{\prime}$ in (30) can be estimated as

$$
E_{3}^{\prime} \ll \frac{T_{3} T_{4}}{K^{\prime}}=\frac{Q^{2}}{\delta_{3} \delta_{4} K^{\prime} K_{3} K_{4}} .
$$

3.4. A counting lemma. For fixed $q_{1}, q_{2}, k_{3}, k_{4}$, our next goal is to estimate the inner sum of the main term of $\sum_{3}$ from (30), which can be written in the simpler form

$$
\sum_{4}=\sum_{\substack{m \in \mathbf{I}, n \in \mathbf{J},(m, n)=(m n, \delta)=1, \frac{b m m}{q} \in(\alpha, \beta]}} 1,
$$

with $\mathbf{I}=\left(\left(k_{3}-1\right) T_{3}, k_{3} T_{3}\right] \subset\left(0, Q / \delta_{3}\right], \mathbf{J}=\left(\left(k_{4}-1\right) T_{4}, k_{4} T_{4}\right] \subset\left(0, Q / \delta_{4}\right]$,

$$
q=a q_{1} q_{2} \leqq Q^{2}
$$

$\alpha=\frac{\left(k^{\prime}-1\right) T^{\prime}}{q}, \beta=\frac{k^{\prime} T^{\prime}}{q}, m=q_{3}, n=q_{4}$ where $b$ is a fixed integer satisfying $(b, q)=1$. Here for an integer $x$ such that $(x, q)=1$, we denote by $\bar{x}$ the multiplicative inverse of $x$ modulo $q$, i.e. $0<\bar{x}<q$ and $\bar{x} x \equiv 1(\bmod q)$.

3.4.1. First step. Defining the set

$$
V:=\left\{x_{m, n}=\frac{\overline{b m n}}{q} \mid m \in \mathbf{I}, n \in \mathbf{J},(m, n)=(m n, q \delta)=1\right\}
$$

we have

$$
\sum_{4}=\#(V \cap(\alpha, \beta])
$$

We will obtain the formula

$$
\begin{aligned}
\sum_{4} & =\#(V \cap(\alpha, \beta])=\frac{6 T_{3} T_{4}}{\pi^{2} K^{\prime}} \prod_{p \mid \delta a q_{1} q_{2}}\left(1-\frac{2}{p+1}\right)+E_{4} \\
& =\frac{6}{\pi^{2} \delta_{3} \delta_{4}} \frac{Q^{2}}{K_{3} K_{4} K^{\prime}} \prod_{p \mid \delta q_{1} q_{2}}\left(1-\frac{2}{p+1}\right)+E_{4}
\end{aligned}
$$

where the error term $E_{4}$ is to be estimated later. To this end first note that

$$
\begin{aligned}
\# V=\sum_{\substack{m \in \mathbf{I}, n \in \mathbf{J},(m, n)=(m n, q \delta)=1}} 1=\sum_{\substack{m \in \mathbf{I},(m, q \delta)=1}} \sum_{\substack{n \in \mathbf{J},(n, m q \delta)=1}} 1=\sum_{\substack{m \in \mathbf{I},(m, q \delta)=1}} \sum_{n \in \mathbf{J}} \sum_{\substack{d|n, d| m q \delta}} \mu(d) \\
=\sum_{\substack{m \in \mathbf{I},(m, q \delta)=1}} \sum_{d \mid m q \delta} \mu(d) \sum_{\substack{d \mid n, n \in \mathbf{J}}} 1=\sum_{\substack{m \in \mathbf{I},(m, q \delta)=1}} \sum_{d \mid m q \delta} \mu(d)\left(\frac{|\mathbf{J}|}{d}+O(1)\right)
\end{aligned}
$$




$$
\begin{aligned}
& =|\mathbf{J}| \sum_{\substack{m \in \mathbf{I},(m, q \delta)=1}} \sum_{d \mid m q \delta} \frac{\mu(d)}{d}+O_{\eta}\left(|\mathbf{I}| Q^{\eta}\right)=|\mathbf{J}| \sum_{\substack{m \in \mathbf{I},(m, q \delta)=1}} \frac{\varphi(m q \delta)}{m q \delta}+O_{\eta}\left(|\mathbf{I}| Q^{\eta}\right) \\
& =|\mathbf{J}| \frac{\varphi(q \delta)}{q \delta} \sum_{\substack{m \in \mathbf{I},(m, q \delta)=1}} \frac{\varphi(m)}{m}+O_{\eta}\left(|\mathbf{I}| Q^{\eta}\right) .
\end{aligned}
$$

We observe that

$$
\begin{aligned}
\sum_{\substack{m \in \mathbf{I},(m, q \delta)=1}} \frac{\varphi(m)}{m} & =\sum_{\substack{m \in \mathbf{I},(m, q \delta)=1}} \sum_{d \mid m} \frac{\mu(d)}{d}=\sum_{\begin{array}{c}
d \leqq Q, \\
(d, q \delta)=1
\end{array}} \frac{\mu(d)}{d} \sum_{\begin{array}{c}
d \mid m, m \in \mathbf{I}, \\
(m, q \delta)=1
\end{array}} 1 \\
& =\sum_{\substack{d \leqq Q,(d, q \delta)=1}} \frac{\mu(d)}{d} \sum_{\substack{m^{\prime} \in \frac{\mathbf{I}}{d},\left(m^{\prime}, q \delta\right)=1}} 1 .
\end{aligned}
$$

Recall the elementary result that if the function $f \in C^{1}(\mathbb{R})$ has compact support, $\mathbf{I}$ is a finite interval, $A$ is a fixed positive integer and $l$ an integer, then

$$
\left|\sum_{\substack{l \in \mathbf{I} \\(l, A)=1}} f(l)-\frac{\varphi(A)}{A} \int_{\mathbf{I}} f(x) \mathrm{d} x\right| \leqq \sigma_{0}(A)\left(\|D f\|_{\infty}|\mathbf{I}|+2\|f\|_{\infty}\right),
$$

where the number of divisor functions satisfies

$$
\sigma_{0}(A)=\sum_{d \mid A} 1 \ll_{\varepsilon} A^{\varepsilon}
$$

for every fixed $\varepsilon>0$. Using this result we have

$$
\begin{aligned}
\sum_{\substack{m \in \mathbf{I},(m, q \delta)=1}} \frac{\varphi(m)}{m} & =\sum_{\substack{d \leqq Q,(d, q \delta)=1}} \frac{\mu(d)}{d}\left(\frac{\varphi(q \delta)}{q \delta} \int_{\frac{1}{d}} 1 \mathrm{~d} t+O_{\eta}\left((q \delta)^{\eta}\right)\right) \\
& =\sum_{\substack{d \leqq Q,(d, q \delta)=1}} \frac{\mu(d)}{d}\left(\frac{\varphi(q \delta)}{q \delta} \frac{|\mathbf{I}|}{d}+O_{\eta}\left(Q^{\eta}\right)\right) \\
& =\frac{\varphi(q \delta)}{q \delta}|\mathbf{I}| \sum_{\substack{d \leqq Q,(d, q \delta)=1}} \frac{\mu(d)}{d^{2}}+O_{\eta}\left(Q^{\eta}\right) .
\end{aligned}
$$

Completing the convergent sum above gives

$$
\begin{aligned}
\sum_{\substack{d \leq Q,(d, q \delta)=1}} \frac{\mu(d)}{d^{2}}=\sum_{\substack{d \geqq 1,(d, q \delta)=1}} \frac{\mu(d)}{d^{2}}-\sum_{\substack{d>Q,(d, q \delta)=1}} \frac{\mu(d)}{d^{2}} \\
=\frac{\prod_{p}\left(1-\frac{1}{p^{2}}\right)}{\prod_{p \mid q \delta}\left(1-\frac{1}{p^{2}}\right)}+O\left(\frac{1}{Q}\right) .
\end{aligned}
$$


Since

$$
\prod_{p \text { prime }}\left(1-\frac{1}{p^{2}}\right)=\frac{6}{\pi^{2}},
$$

and $|\mathbf{I}|<Q$, we obtain

$$
\sum_{\substack{m \in \mathbf{I},(m, q \delta)=1}} \frac{\varphi(m)}{m}=\frac{\varphi(q \delta)}{q \delta}|\mathbf{I}| \frac{6}{\pi^{2}} \frac{1}{\prod_{p \mid q \delta}\left(1-\frac{1}{p^{2}}\right)}+O_{\eta}\left(Q^{\eta}\right) .
$$

Inserting this into the above expression for \# $V$ finally gives

$$
\begin{aligned}
\# V & =|\mathbf{J}| \frac{\varphi(q \delta)}{q \delta}\left(\frac{\varphi(q \delta)}{q \delta}|\mathbf{I}| \frac{6}{\pi^{2}} \frac{1}{\prod_{p \mid q \delta}\left(1-\frac{1}{p^{2}}\right)}+O_{\eta}\left(Q^{\eta}\right)\right)+O_{\eta}\left(|\mathbf{I}| Q^{\eta}\right) \\
& =|\mathbf{I}| \cdot|\mathbf{J}| \frac{6}{\pi^{2}} \frac{\prod_{p \mid q \delta}\left(1-\frac{1}{p}\right)^{2}}{\prod_{p \mid q \delta}\left(1-\frac{1}{p^{2}}\right)}+O_{\eta}\left(|\mathbf{J}| Q^{\eta}\right)+O_{\eta}\left(|\mathbf{I}| Q^{\eta}\right) \\
& =\frac{6|\mathbf{I}| \cdot|\mathbf{J}|}{\pi^{2}} \prod_{p \mid q \delta}\left(1-\frac{2}{p+1}\right)+O_{\eta}\left((|\mathbf{I}|+|\mathbf{J}|) \cdot Q^{\eta}\right) .
\end{aligned}
$$

3.4.2. Second step. By the Erdös-Turán inequality ([31]),

$$
\begin{aligned}
|\#(V \cap(\alpha, \beta])-(\beta-\alpha) \# V| & \ll \frac{\# V}{L}+\sum_{1 \leqq K \leqq L} \frac{1}{K}\left|\sum_{x_{m, n} \in V} e\left(K x_{m, n}\right)\right| \\
& \ll \frac{|\mathbf{I}| \cdot|\mathbf{J}|}{L}+\sum_{1 \leqq K \leqq L} \frac{S_{K}}{K} .
\end{aligned}
$$

Here $L$ is a large real number to be chosen later and

$$
S_{K}=\left|\sum_{x_{m, n} \in V} e\left(K x_{m, n}\right)\right|
$$

Define, for $1 \leqq K \leqq L$,

$$
S(\mathbf{I}, \mathbf{J}, \delta, q, l)=\sum_{\substack{m \in \mathbf{I},(m, q \delta)=1}} \sum_{\substack{n \in \mathbf{J},(n, q \delta)=1}} e\left(\frac{l \bar{m} \bar{n}}{q}\right)
$$

and note that taking $x_{m, n}=\frac{\bar{b} \bar{m} \bar{n}}{q}, S_{K}$ can be rewritten as 


$$
\begin{aligned}
S_{K} & =\left|\sum_{\substack{m \in \mathbf{I},(m, q \delta)=1}} \sum_{\substack{n \in \mathbf{J},(n, m q \delta)=1}} e\left(\frac{K \bar{b} \bar{m} \bar{n}}{q}\right)\right|=\left|\sum_{\substack{m \in \mathbf{I},(m, q \delta)=1}} \sum_{\substack{n \in \mathbf{J},(n, q \delta)=1}} e\left(\frac{K \bar{b} \bar{m} \bar{n}}{q}\right) \sum_{\substack{d|n, d| m}} \mu(d)\right| \\
& \left.=\mid \sum_{\substack{d \leqq Q,(d, q \delta)=1}} \mu(d) \sum_{\substack{m \in \frac{\mathbf{I}}{d},(m, q \delta)=1}} \sum_{\substack{n \in \frac{\mathbf{J}}{d},(n, q \delta)=1}} e \frac{K \overline{b d^{2}} \bar{m} \bar{n}}{q}\right) \mid \\
& =\left|\sum_{\substack{d \leqq Q,(d, q \delta)=1}} \mu(d) S\left(\frac{\mathbf{I}}{d}, \frac{\mathbf{J}}{d}, \delta, q, K \overline{b d^{2}}\right)\right|
\end{aligned}
$$

We use the trivial estimate

$$
\begin{aligned}
\left|S\left(\frac{\mathbf{I}}{d}, \frac{\mathbf{J}}{d}, \delta, q, K \overline{b d^{2}}\right)\right| & \leqq\left(\frac{|\mathbf{I}|}{d}+1\right)\left(\frac{|\mathbf{J}|}{d}+1\right) \\
& =\frac{|\mathbf{I}| \cdot|\mathbf{J}|}{d^{2}}+\frac{|\mathbf{I}|}{d}+\frac{|\mathbf{J}|}{d}+1,
\end{aligned}
$$

and let $R$ be a real number to be chosen later with $0<R<|\mathbf{I}|$, to deduce that

$$
\begin{aligned}
\sum_{\substack{R<d \leqq Q,(d, q \delta)=1}} \mu(d) S\left(\frac{\mathbf{I}}{d}, \frac{\mathbf{J}}{d}, \delta, q, K \overline{b d^{2}}\right) \mid & \leqq \sum_{R<d \leqq Q}\left(\frac{|\mathbf{I}| \cdot|\mathbf{J}|}{d^{2}}+\frac{|\mathbf{I}|}{d}+\frac{|\mathbf{J}|}{d}+1\right) \\
& \leqq|\mathbf{I}| \cdot|\mathbf{J}| \sum_{d>R} \frac{1}{d^{2}}+(|\mathbf{I}|+|\mathbf{J}|) \sum_{d>R} \frac{1}{d}+Q \\
& \ll \frac{|\mathbf{I}| \cdot|\mathbf{J}|}{R}+(|\mathbf{I}|+|\mathbf{J}|) \log Q+Q .
\end{aligned}
$$

The main difficulty comes from small values of $d$, namely if $1 \leqq d \leqq R$, then we write

$$
\left.S=S\left(\frac{\mathbf{I}}{d}, \frac{\mathbf{J}}{d}, \delta, q, K \overline{b d^{2}}\right)=\sum_{\substack{u \in \frac{\mathbf{I}}{d},(u, q \delta)=1}} \sum_{\substack{v \in \frac{\mathbf{J}}{d},(v, q \delta)=1}} e \frac{K \overline{b d^{2}} \bar{u} \bar{v}}{q}\right) .
$$

Applying Hölder's inequality and noting that $(u, q \delta)=1$ implies $(u, q)=1$, we have

$$
\left.|S|^{4} \leqq\left(\sum_{\substack{u \in \frac{1}{d},(u, q \delta)=1}} 1\right)^{3} \cdot \sum_{\substack{u \in \frac{\mathrm{I}}{d},(u, q)=1}}\left|\sum_{\substack{v \in \frac{\mathrm{J}}{d},(v, q \delta)=1}} e\left(\frac{K \overline{b d^{2}} \bar{u} \bar{v}}{q}\right)\right|^{4}\right) .
$$

We will distinguish two cases, namely that $q \leqq|\mathbf{I}| / d$ and $q>|\mathbf{I}| / d$. First assuming $q \leqq|\mathbf{I}| / d$, observe that there are $\ll|\mathbf{I}| / d q$ consecutive intervals of length $q$ covering all of the interval $\mathbf{I} / d$. Therefore we obtain from (36) that

$$
\left.|S|^{4} \ll\left(\frac{|\mathbf{I}|}{d}\right)^{4} \frac{1}{q} \sum_{\substack{v_{1}, v_{2}, v_{3}, v_{4} \in \frac{\mathrm{J}}{\bar{d}},\left(v_{i}, q \delta\right)=1}} \sum_{\substack{1 \leqq u \leqq q,(u, q)=1}} e \frac{K \overline{b d^{2}} \bar{u}\left(\overline{v_{1}}+\overline{v_{2}}-\overline{v_{3}}-\overline{v_{4}}\right)}{q}\right) .
$$


As $u$ runs through a reduced residue system modulo $q$, then so is $\overline{b d^{2}} \bar{u}$, so that (37) becomes

$$
\begin{aligned}
& |S|^{4} \ll\left(\frac{|\mathbf{I}|}{d}\right)^{4} \frac{1}{q} \sum_{\substack{v_{1}, v_{2}, v_{3}, v_{4} \in \frac{\mathbf{J}}{d},\left(v_{i}, q \delta\right)=1}} \sum_{\substack{1 \leqq u \leqq q \\
(u, q)=1}} e\left(\frac{K u\left(\overline{v_{1}}+\overline{v_{2}}-\overline{v_{3}}-\overline{v_{4}}\right)}{q}\right) \\
& =\left(\frac{|\mathbf{I}|}{d}\right)^{4} \frac{1}{q} \sum_{\substack{v_{1}, v_{2}, v_{3}, v_{4} \in \frac{\mathbf{J}}{d},\left(v_{i}, q \delta\right)=1}} \sum_{1 \leqq u \leqq q} e\left(\frac{K u\left(\overline{v_{1}}+\overline{v_{2}}-\overline{v_{3}}-\overline{v_{4}}\right)}{q}\right) \sum_{\substack{s|u, s| q}} \mu(s) \\
& =\left(\frac{|\mathbf{I}|}{d}\right)^{4} \frac{1}{q} \sum_{s \mid q} \mu(s) \sum_{\substack{v_{1}, v_{2}, v_{3}, v_{4} \in \frac{\mathrm{J}}{d}, 1 \leqq t \leqq \frac{q}{s} \\
\left(v_{i}, q \delta\right)=1}} \sum_{\substack{\frac{K}{s} \\
s}}\left(\frac{\left.K t \overline{v_{1}}+\overline{v_{2}}-\overline{v_{3}}-\overline{v_{4}}\right)}{\frac{q}{s}}\right) .
\end{aligned}
$$

Using the fact that

$$
\sum_{1 \leqq t \leqq q} e\left(\frac{K t\left(\overline{v_{1}}+\overline{v_{2}}-\overline{v_{3}}-\overline{v_{4}}\right)}{\frac{q}{s}}\right)=\frac{q}{S}
$$

when

$$
K\left(\overline{v_{1}}+\overline{v_{2}}-\overline{v_{3}}-\overline{v_{4}}\right) \equiv 0\left(\bmod \frac{q}{s}\right)
$$

and zero otherwise, we have from (38) that

$$
\begin{aligned}
|S|^{4} \ll & \left(\frac{|\mathbf{I}|}{d}\right)^{4} \sum_{s \mid q} \frac{1}{s} \\
& \times \#\left\{\begin{array}{l|l}
\left(v_{1}, v_{2}, v_{3}, v_{4}\right) & \begin{array}{l}
v_{i} \in \frac{\mathbf{J}}{d},\left(v_{i}, q \delta\right)=1, \\
K\left(\overline{v_{1}}+\overline{v_{2}}-\overline{v_{3}}-\overline{v_{4}}\right) \equiv 0\left(\bmod \frac{q}{s}\right)
\end{array}
\end{array} .\right.
\end{aligned}
$$

A similar argument for the case $q>\frac{|\mathbf{I}|}{d}$ gives

$$
\begin{aligned}
& |S|^{4} \ll\left(\frac{|\mathbf{I}|}{d}\right)^{3} q \sum_{s \mid q} \frac{1}{s} \\
& \times \#\left\{\begin{array}{l|l}
\left(v_{1}, v_{2}, v_{3}, v_{4}\right) & \begin{array}{l}
v_{i} \in \frac{\mathbf{J}}{d},\left(v_{i}, q \delta\right)=1, \\
K\left(\overline{v_{1}}+\overline{v_{2}}-\overline{v_{3}}-\overline{v_{4}}\right) \equiv 0\left(\bmod \frac{q}{s}\right)
\end{array}
\end{array} .\right.
\end{aligned}
$$

Since $\frac{|\mathbf{I}|}{d}<Q$ and $q \leqq Q^{2},(39)$ and (40) can be combined under the single estimate 
(42)

$$
\begin{aligned}
& |S|^{4} \ll\left(\frac{|\mathbf{I}|}{d}\right)^{3} Q^{2} \sum_{s \mid q} \frac{1}{s} \\
& \times \#\left\{\begin{array}{l|l}
\left(v_{1}, v_{2}, v_{3}, v_{4}\right) & \begin{array}{l}
v_{i} \in \frac{\mathbf{J}}{d},\left(v_{i}, q \delta\right)=1, \\
K\left(\overline{v_{1}}+\overline{v_{2}}-\overline{v_{3}}-\overline{v_{4}}\right) \equiv 0\left(\bmod \frac{q}{s}\right)
\end{array}
\end{array} .\right.
\end{aligned}
$$

We need to control the number of all admissible tuples $\left(v_{1}, v_{2}, v_{3}, v_{4}\right)$ appearing in (41). Although it is possible to obtain reasonable upper bounds for individual $q$, the quality of these bounds would not be good enough to arrive at an error term which is $o\left(Q^{4}\right)$. Therefore we prefer to average over all $q \leqq Q^{2}$. Clearly the condition

$$
K\left(\overline{v_{1}}+\overline{v_{2}}-\overline{v_{3}}-\overline{v_{4}}\right) \equiv 0\left(\bmod \frac{q}{s}\right)
$$

for $s \mid q$ implies

$$
K\left(v_{1} v_{2}\left(v_{3}+v_{4}\right)-v_{3} v_{4}\left(v_{1}+v_{2}\right)\right) \equiv 0\left(\bmod \frac{q}{s}\right)
$$

Consequently we have

$$
\begin{aligned}
& \sum_{q \leqq Q^{2}} \sum_{s \mid q} \frac{1}{s} \#\left\{\begin{array}{l|l}
\left(v_{1}, v_{2}, v_{3}, v_{4}\right) & \begin{array}{l}
v_{i} \in \frac{\mathbf{J}}{d},\left(v_{i}, q \delta\right)=1, \\
K\left(\overline{v_{1}}+\overline{v_{2}}-\overline{v_{3}}-\overline{v_{4}}\right) \equiv 0\left(\bmod \frac{q}{s}\right)
\end{array}
\end{array}\right\}
\end{aligned}
$$

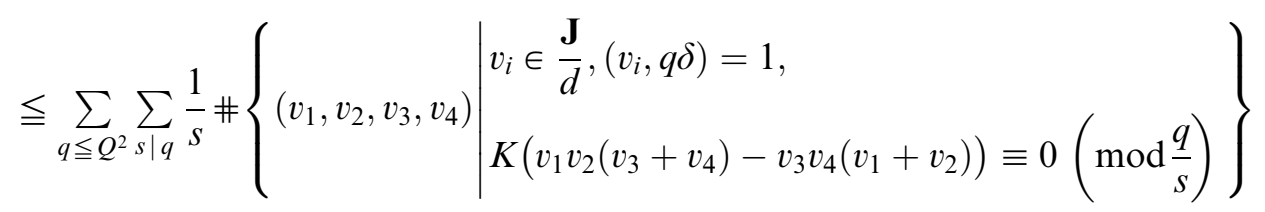

$$
\begin{aligned}
& =\sum_{s \leqq Q^{2}} \frac{1}{S} \sum_{\substack{q \leqq Q^{2}, s \mid q}} \#\left\{\left(v_{1}, v_{2}, v_{3}, v_{4}\right) \mid \begin{array}{l}
v_{i} \in \frac{\mathbf{J}}{d},\left(v_{i}, q \delta\right)=1, \\
K\left(v_{1} v_{2}\left(v_{3}+v_{4}\right)-v_{3} v_{4}\left(v_{1}+v_{2}\right)\right) \equiv 0\left(\bmod \frac{q}{s}\right)
\end{array}\right\} .
\end{aligned}
$$

Fixing $s \leqq Q^{2}$ temporarily, we observe that

$$
\begin{aligned}
& \sum_{\substack{q \leqq Q^{2} \\
s \mid q}} \#\left\{\left(v_{1}, v_{2}, v_{3}, v_{4}\right) \mid \begin{array}{l}
v_{i} \in \frac{\mathbf{J}}{d},\left(v_{i}, q \delta\right)=1, \\
K\left(v_{1} v_{2}\left(v_{3}+v_{4}\right)-v_{3} v_{4}\left(v_{1}+v_{2}\right)\right) \equiv 0\left(\bmod \frac{q}{s}\right)
\end{array}\right\} \\
& \leqq \#\left\{\left(q, v_{1}, v_{2}, v_{3}, v_{4}\right) \mid \begin{array}{l}
q \leqq Q^{2}, v_{i} \in \frac{\mathbf{J}}{d}, s \mid q, \\
K\left(v_{1} v_{2}\left(v_{3}+v_{4}\right)-v_{3} v_{4}\left(v_{1}+v_{2}\right)\right) \equiv 0\left(\bmod \frac{q}{s}\right)
\end{array}\right\} .
\end{aligned}
$$


In order to find a useful upper bound for the number of admissible tuples $\left(q, v_{1}, v_{2}, v_{3}, v_{4}\right)$, we have to distinguish two cases. First of all, if $v_{1} v_{2}\left(v_{3}+v_{4}\right) \neq v_{3} v_{4}\left(v_{1}+v_{2}\right)$, then using the fact that

$$
\frac{q}{s} \mid K\left(v_{1} v_{2}\left(v_{3}+v_{4}\right)-v_{3} v_{4}\left(v_{1}+v_{2}\right)\right)
$$

and

$$
0 \neq\left|K\left(v_{1} v_{2}\left(v_{3}+v_{4}\right)-v_{3} v_{4}\left(v_{1}+v_{2}\right)\right)\right| \leqq 2 Q^{5}
$$

it follows that the number of such integers $q / s$ is bounded by the number of divisors of $K\left(v_{1} v_{2}\left(v_{3}+v_{4}\right)-v_{3} v_{4}\left(v_{1}+v_{2}\right)\right)$, which is $\ll_{\eta} Q^{\eta}$ for $\eta>0$. Since $s$ is fixed, for each tuple $\left(v_{1}, v_{2}, v_{3}, v_{4}\right)$, the number of admissible $q$ is again $\ll_{\eta} Q^{\eta}$. In conclusion, the number of all admissible tuples $\left(q, v_{1}, v_{2}, v_{3}, v_{4}\right)$ is

$$
\ll_{\eta}\left(\frac{|\mathbf{J}|}{d}\right)^{4} Q^{\eta} .
$$

In the other case, if $v_{1} v_{2}\left(v_{3}+v_{4}\right)=v_{3} v_{4}\left(v_{1}+v_{2}\right)$, then fix $v_{3}, v_{4} \in \mathbf{J} / d \cap \mathbb{Z}$ and put

$$
\frac{1}{v_{3}}+\frac{1}{v_{4}}=\frac{a}{b}
$$

where $a, b$ are integers with $(a, b)=1,|a|,|b| \leqq Q^{2}$. We consider solutions of the equation

$$
\frac{1}{v_{1}}+\frac{1}{v_{2}}=\frac{a}{b}
$$

for $v_{1}, v_{2} \in \mathbf{J} / d \cap \mathbb{Z}$. Equivalently, we may write $b\left(v_{1}+v_{2}\right)=a v_{1} v_{2}$. Taking $\left(v_{1}, v_{2}\right)=\tilde{d}$, $v_{1}=\tilde{d} n, v_{2}=\tilde{d} m$ with $(m, n)=1$, gives

$$
b(m+n)=a \tilde{d} m n
$$

Using $(m, m+n)=(n, m+n)=1$, we have $m|b, n| b$, and the number of such pairs $(m, n)$ is $\ll_{\eta} b^{\eta} \ll_{\eta} Q^{\eta}$. Since $\tilde{d} \mid b(m+n)$, the number of $\tilde{d}$ for fixed $m, n$ is $\ll_{\eta} Q^{\eta}$. Therefore for fixed $v_{3}, v_{4}$, the number of such pairs $\left(v_{1}, v_{2}\right)$ is also $\ll_{\eta} Q^{\eta}$. Observing that there are at most $Q^{2}$ choices for $q$ and at most $\ll(|\mathbf{J}| / d)^{2}$ choices for the pairs $\left(v_{3}, v_{4}\right)$, the number of admissible tuples $\left(q, v_{1}, v_{2}, v_{3}, v_{4}\right)$ in this case is

$$
\ll_{\eta}\left(\frac{|\mathbf{J}|}{d}\right)^{2} Q^{2+\eta} .
$$

Using $|\mathbf{J}| / d<Q$ and combining the two cases, we see that

$$
\#\left\{\begin{array}{c|c}
\left.\left(q, v_{1}, v_{2}, v_{3}, v_{4}\right) \mid \begin{array}{l}
q \leqq Q^{2}, v_{i} \in \frac{\mathbf{J}}{d}, s \mid q \\
K\left(v_{1} v_{2}\left(v_{3}+v_{4}\right)-v_{3} v_{4}\left(v_{1}+v_{2}\right)\right) \equiv 0\left(\bmod \frac{q}{s}\right)
\end{array}\right\} \\
\ll_{\eta}\left(\frac{|\mathbf{J}|}{d}\right)^{2} Q^{2+\eta}
\end{array}\right.
$$


for $\eta>0$. Combining (42), (43) and (44), we deduce

$$
\begin{aligned}
& \sum_{q \leqq Q^{2}} \sum_{s \mid q} \frac{1}{s} \#\left\{\begin{array}{l|l}
\left(v_{1}, v_{2}, v_{3}, v_{4}\right) & \begin{array}{l}
v_{i} \in \frac{\mathbf{J}}{d},\left(v_{i}, q \delta\right)=1, \\
K\left(\overline{v_{1}}+\overline{v_{2}}-\overline{v_{3}}-\overline{v_{4}}\right) \equiv 0\left(\bmod \frac{q}{s}\right)
\end{array}
\end{array}\right\} \\
& \ll_{\eta}\left(\frac{|\mathbf{J}|}{d}\right)^{2} Q^{2+\eta} \sum_{s \leqq Q^{2}} \frac{1}{S} \ll_{\eta}\left(\frac{|\mathbf{J}|}{d}\right)^{2} Q^{2+\eta} \log Q \ll_{\eta}\left(\frac{|\mathbf{J}|}{d}\right)^{2} Q^{2+\eta}
\end{aligned}
$$

for $\eta>0$. Let $0<\sigma<1$ be a parameter which we will fix later. As a result of (45), the number of $q \leqq Q^{2}$ such that

$$
\sum_{s \mid q} \frac{1}{s} \#\left\{\begin{array}{l|l}
\left(v_{1}, v_{2}, v_{3}, v_{4}\right) & \begin{array}{l}
v_{i} \in \frac{\mathbf{J}}{d},\left(v_{i}, q \delta\right)=1, \\
K\left(\overline{v_{1}}+\overline{v_{2}}-\overline{v_{3}}-\overline{v_{4}}\right) \equiv 0\left(\bmod \frac{q}{s}\right)
\end{array}
\end{array}\right\}>\left(\frac{|\mathbf{J}|}{d}\right)^{3-\sigma}
$$

is

$$
\ll_{\eta} \frac{Q^{2+\eta}}{\left(\frac{|\mathbf{J}|}{d}\right)^{1-\sigma}}
$$

Let $B_{\sigma, K, \frac{\mathrm{J}}{d}}(Q)$ be the set of all $q \leqq Q^{2}$ such that (46) holds. Clearly we have

$$
\left|B_{\sigma, K, \frac{\mathrm{J}}{d}}(Q)\right| \ll_{\eta} \frac{Q^{2+\eta}}{\left(\frac{|\mathbf{J}|}{d}\right)^{1-\sigma}} .
$$

Also let $G_{\sigma, K, \frac{\mathrm{J}}{d}}(Q)$ be the complementary set of $B_{\sigma, K, \frac{\mathrm{J}}{d}}(Q)$ in $\left(0, Q^{2}\right]$. If $q \leqq Q^{2}$ and $q \in G_{\sigma, K, \frac{\mathrm{J}}{\mathrm{d}}}(Q)$, then it follows from (41) that

$$
|S|^{4} \leqq\left(\frac{|\mathbf{I}|}{d}\right)^{3} Q^{2}\left(\frac{|\mathbf{J}|}{d}\right)^{3-\sigma}
$$

and consequently that

$$
|S| \leqq Q^{\frac{1}{2}}\left(\frac{|\mathbf{I}|}{d}\right)^{\frac{3}{4}}\left(\frac{|\mathbf{J}|}{d}\right)^{\frac{3-\sigma}{4}}=Q^{\frac{1}{2}} \frac{|\mathbf{I}|^{\frac{3}{4}} \cdot|\mathbf{J}|^{\frac{3-\sigma}{4}}}{d^{\frac{6-\sigma}{4}}} .
$$

Therefore if $q \in \bigcap_{d \leqq R} G_{\sigma, K, \frac{\mathrm{J}}{d}}(Q)$, then

$$
\begin{aligned}
& \left|\sum_{\substack{d \leqq R,(d, q \delta)=1}} \mu(d) S\left(\frac{\mathbf{I}}{d}, \frac{\mathbf{J}}{d}, \delta, q, K \overline{b d^{2}}\right)\right| \leqq \sum_{d \leqq R,} Q^{\frac{1}{2}} \frac{|\mathbf{I}|^{\frac{3}{4}} \cdot|\mathbf{J}|^{\frac{3-\sigma}{4}}}{d^{\frac{6-\sigma}{4}}} \\
& \ll Q^{\frac{1}{2}}|\mathbf{I}|^{\frac{3}{4}} \cdot|\mathbf{J}|^{\frac{3-\sigma}{4}},
\end{aligned}
$$


since

$$
\frac{6-\sigma}{4}>\frac{5}{4}>1
$$

In conclusion, for

$$
q \in \bigcap_{1 \leqq K \leqq L} \bigcap_{d \leqq R} G_{\sigma, K, \frac{\mathrm{J}}{d}}(Q),
$$

one has from (34), (35) and (49) that

$$
\begin{aligned}
S_{K} & \leqq\left|\sum_{\substack{R<d \leqq Q,(d, q \delta)=1}} \mu(d) S\left(\frac{\mathbf{I}}{d}, \frac{\mathbf{J}}{d}, \delta, q, K \overline{b d^{2}}\right)\right|+\left|\sum_{\substack{d \leqq R,(d, q \delta)=1}} \mu(d) S\left(\frac{\mathbf{I}}{d}, \frac{\mathbf{J}}{d}, \delta, q, K \overline{b d^{2}}\right)\right| \\
& \ll\left(\frac{|\mathbf{I}| \cdot|\mathbf{J}|}{R}+(|\mathbf{I}|+|\mathbf{J}|) \log Q+Q\right)+\left(Q^{\left.\frac{1}{2}|\mathbf{I}|^{\frac{3}{4}} \cdot|\mathbf{J}|^{\frac{3-\sigma}{4}}\right) .}\right.
\end{aligned}
$$

Finally we obtain for such $q$ that

$$
\begin{aligned}
\mid \#( & \cap(\alpha, \beta])-(\beta-\alpha) \# V \mid \ll \frac{|\mathbf{I}| \cdot|\mathbf{J}|}{L}+\sum_{1 \leqq K \leqq L} \frac{S_{K}}{K} \\
& \ll \frac{|\mathbf{I}| \cdot|\mathbf{J}|}{L}+\left(\frac{|\mathbf{I}| \cdot|\mathbf{J}|}{R}+(|\mathbf{I}|+|\mathbf{J}|) \log Q+Q+Q^{\frac{1}{2}}|\mathbf{I}|^{\frac{3}{4}} \cdot|\mathbf{J}|^{\frac{3-\sigma}{4}}\right) \log L .
\end{aligned}
$$

3.4.3. Third step. Recall that

$$
|\mathbf{I}|=T_{3}, \quad|\mathbf{J}|=T_{4}, \quad q=a q_{1} q_{2}, \quad \text { and } \quad \beta-\alpha=\frac{T^{\prime}}{a q_{1} q_{2}}=\frac{1}{K^{\prime}}
$$

Choosing

$$
R=L<T_{3}<Q
$$

we see that

$$
\log R=\log L \leqq \log Q \ll_{\eta} Q^{\eta}
$$

In this way (51) becomes

$$
|\#(V \cap(\alpha, \beta])-(\beta-\alpha) \# V| \ll_{\eta}\left(\frac{T_{3} T_{4}}{L}+Q+Q^{\frac{1}{2}} T_{3}^{\frac{3}{4}} \cdot T_{4}^{\frac{3-\sigma}{4}}\right) Q^{\eta}
$$

Recall that

$$
\# V=\frac{6 T_{3} T_{4}}{\pi^{2}} \prod_{p \mid \delta q}\left(1-\frac{2}{p+1}\right)+O_{\eta}\left(\left(T_{3}+T_{4}\right) \cdot Q^{\eta}\right)
$$


and

$$
(\beta-\alpha) \cdot \# V=\frac{6 T_{3} T_{4}}{\pi^{2} K^{\prime}} \prod_{p \mid \delta q}\left(1-\frac{2}{p+1}\right)+O_{\eta}\left(\frac{T_{3}+T_{4}}{K^{\prime}} \cdot Q^{\eta}\right) .
$$

Since $K_{3} T_{3}=Q / \delta_{3}, K_{4} T_{4}=Q / \delta_{4}$ and $a \mid \delta$, from (32) we obtain, as promised in the beginning of Section 3.4, that

$$
\begin{aligned}
\sum_{4} & =\#(V \cap(\alpha, \beta])=\frac{6 T_{3} T_{4}}{\pi^{2} K^{\prime}} \prod_{p \mid \delta a q_{1} q_{2}}\left(1-\frac{2}{p+1}\right)+E_{4} \\
& =\frac{6}{\pi^{2} \delta_{3} \delta_{4}} \frac{Q^{2}}{K_{3} K_{4} K^{\prime}} \prod_{p \mid \delta q_{1} q_{2}}\left(1-\frac{2}{p+1}\right)+E_{4},
\end{aligned}
$$

where the error term $E_{4}$ is estimated as

$$
\begin{aligned}
E_{4} & \ll_{\eta} \frac{T_{3}+T_{4}}{K^{\prime}} \cdot Q^{\eta}+\left(\frac{T_{3} T_{4}}{L}+Q+Q^{\frac{1}{2}} T_{3}^{\frac{3}{4}} \cdot T_{4}^{\frac{3-\sigma}{4}}\right) Q^{\eta} \\
& \ll_{\eta}\left(\frac{T_{3} T_{4}}{L}+Q+Q^{\frac{1}{2}} T_{3}^{\frac{3}{4}} \cdot T_{4}^{\frac{3-\sigma}{4}}\right) Q^{\eta} .
\end{aligned}
$$

\subsection{Estimation of error terms.}

3.5.1. First step. Denoting

$$
U_{\sigma}:=\bigcap\left\{G_{\sigma, K, \frac{\mathrm{J}}{d}}(Q) \mid 1 \leqq d \leqq L, 1 \leqq K \leqq L, 1 \leqq k_{4} \leqq K_{4}, \mathbf{J}=\left(\left(k_{4}-1\right) T_{4}, k_{4} T_{4}\right]\right\},
$$

for $a q_{1} q_{2} \in U_{\sigma}$, and gathering $\sum_{3}, \sum_{4}$ from (30), (52) we have

$$
\begin{aligned}
\sum_{3} & =\sum_{1 \leqq k^{\prime} \leqq K^{\prime}} f\left(\frac{k^{\prime}}{K^{\prime}}\right)\left(\frac{6 Q^{2}}{\pi^{2} \delta_{3} \delta_{4} K_{3} K_{4} K^{\prime}} \prod_{p \mid \delta q_{1} q_{2}}\left(1-\frac{2}{p+1}\right)+E_{4}\right)+E_{3}^{\prime} \\
& =\frac{6 Q^{2}}{\pi^{2} \delta_{3} \delta_{4} K_{3} K_{4}} \prod_{p \mid \delta q_{1} q_{2}}\left(1-\frac{2}{p+1}\right) \frac{1}{K^{\prime}} \sum_{1 \leqq k^{\prime} \leqq K^{\prime}} f\left(\frac{k^{\prime}}{K^{\prime}}\right)+E_{3}^{\prime \prime},
\end{aligned}
$$

where

$$
E_{3}^{\prime \prime} \ll K^{\prime} E_{4}+E_{3}^{\prime},
$$

and using the estimates for $E_{4}$ and $E_{3}^{\prime}$ from (31), (53) we get

$$
E_{3}^{\prime \prime} \ll_{\eta}\left(\frac{T_{3} T_{4}}{L}+Q+Q^{\frac{1}{2}} T_{3}^{\frac{3}{4}} \cdot T_{4}^{\frac{3-\sigma}{4}}\right) K^{\prime} Q^{\eta}+\frac{Q^{2}}{\delta_{3} \delta_{4} K_{3} K_{4} K^{\prime}} .
$$

Recall that if the function $f \in C^{1}(\mathbb{R})$ and $K$ is any positive integer, then

$$
\left|\frac{1}{K} \sum_{k=1}^{K} f\left(\frac{k}{K}\right)-\int_{0}^{1} f(x) \mathrm{d} x\right| \leqq \frac{\|D f\|_{\infty}}{K}
$$


Using this elementary result and (16), one has

$$
\frac{1}{K^{\prime}} \sum_{1 \leqq k^{\prime} \leqq K^{\prime}} f\left(\frac{k^{\prime}}{K^{\prime}}\right)=\int_{0}^{1} f(x) \mathrm{d} x+O\left(\frac{1}{K^{\prime}}\right),
$$

so that

$$
\sum_{3}=\frac{6 Q^{2}}{\pi^{2} \delta_{3} \delta_{4} K_{3} K_{4}} \prod_{p}\left(1-\frac{2}{p+1}\right) \int_{0}^{1} f(x) \mathrm{d} x+E_{3},
$$

where we can estimate $E_{3}$ as

$$
E_{3} \ll E_{3}^{\prime \prime}+\frac{Q^{2}}{\delta_{3} \delta_{4} K_{3} K_{4} K^{\prime}}
$$

3.5.2. Second step. Going back to $\sum_{2}$ from (27) and using the error term, for $a q_{1} q_{2} \in U_{\sigma}$ we have

$$
\begin{aligned}
& \sum_{2}=\sum_{\substack{1 \leqq k_{3} \leqq K_{3}, 1 \leqq k_{4} \leqq K_{4}}} h\left(q_{1}, q_{2}, \frac{Q}{\delta_{3}} \frac{k_{3}}{K_{3}}, \frac{Q}{\delta_{4}} \frac{k_{4}}{K_{4}}\right) \\
& \times\left(\frac{6 Q^{2}}{\pi^{2} \delta_{3} \delta_{4} K_{3} K_{4}} \prod_{p \mid \delta q_{1} q_{2}}\left(1-\frac{2}{p+1}\right) \int_{0}^{1} f(x) \mathrm{d} x+E_{3}\right)+E_{2}^{\prime} \\
& =\frac{6 Q^{2}}{\pi^{2} \delta_{3} \delta_{4}} \int_{0}^{1} f(x) \mathrm{d} x \prod_{p \mid \delta q_{1} q_{2}}\left(1-\frac{2}{p+1}\right) \\
& \times \frac{1}{K_{3} K_{4}} \sum_{\substack{1 \leqq k_{3} \leqq K_{3}, 1 \leqq k_{4} \leqq K_{4}}} h\left(q_{1}, q_{2}, \frac{Q}{\delta_{3}} \frac{k_{3}}{T_{3}}, \frac{Q}{\delta_{4}} \frac{k_{4}}{T_{4}}\right)+E_{2}^{\prime \prime},
\end{aligned}
$$

where

$$
E_{2}^{\prime \prime} \ll K_{3} K_{4} E_{3}+E_{2}^{\prime} \ll K_{3} K_{4} E_{3}+\left(T_{3}+T_{4}\right) Q .
$$

Applying (56) two times to the sum

$$
\frac{1}{K_{3} K_{4}} \sum_{\substack{1 \leqq k_{3} \leqq K_{3}, 1 \leqq k_{4} \leqq K_{4}}} h\left(q_{1}, q_{2}, \frac{Q}{\delta_{3}} \frac{k_{3}}{T_{3}}, \frac{Q}{\delta_{4}} \frac{k_{4}}{T_{4}}\right)
$$

we get

$$
\begin{aligned}
\frac{1}{K_{3} K_{4}} \sum_{\substack{1 \leqq k_{3} \leqq K_{3}, 1 \leqq k_{4} \leqq K_{4}}} h\left(q_{1}, q_{2}, \frac{Q}{\delta_{3}} \frac{k_{3}}{T_{3}}, \frac{Q}{\delta_{4}} \frac{k_{4}}{T_{4}}\right)= & \iint h\left(q_{1}, q_{2}, \frac{Q}{\delta_{3}} z, \frac{Q}{\delta_{4}} w\right) \mathrm{d} z \mathrm{~d} w \\
& +O\left(\frac{1}{K_{3}}+\frac{1}{K_{4}}\right) .
\end{aligned}
$$


Therefore

$$
\begin{aligned}
\sum_{2}= & \frac{6 Q^{2}}{\pi^{2} \delta_{3} \delta_{4}}\left(\int_{0}^{1} f(x) \mathrm{d} x\right) \prod_{p \mid \delta q_{1} q_{2}}\left(1-\frac{2}{p+1}\right) \\
& \times \iint_{[0,1]^{2}} h\left(q_{1}, q_{2}, \frac{Q}{\delta_{3}} z, \frac{Q}{\delta_{4}} w\right) \mathrm{d} z \mathrm{~d} w+E_{2},
\end{aligned}
$$

where

$$
E_{2} \ll Q^{2}\left(\frac{1}{K_{3}}+\frac{1}{K_{4}}\right)+E_{2}^{\prime \prime}
$$

3.5.3. Third step. Now returning to $\sum_{1}$ from (24), we may write it as

$$
\begin{aligned}
\sum_{1}= & \sum_{\substack{q_{1} \leqq Q / \delta_{1}, q_{2} \leqq Q / \delta_{2},\left(q_{1}, q_{2} \delta\right)=1,\left(q_{2}, \delta\right)=1}}\left(\sum_{a q_{1} q_{2} \in U_{\sigma}}+\sum_{a q_{1} q_{2} \notin U_{\sigma}}\right) \\
= & \sum_{1}^{\prime}+\sum_{1}^{\prime \prime} .
\end{aligned}
$$

As we know the complement of $\bar{U}_{\sigma}$ is given by

$$
\begin{aligned}
\bar{U}_{\sigma} & :=\bigcup\left\{B_{\sigma, K, \frac{\mathrm{J}}{d}}(Q) \mid 1 \leqq d \leqq L, 1 \leqq K \leqq L, 1 \leqq k_{4} \leqq K_{4}, \mathbf{J}=\left(\left(k_{4}-1\right) T_{4}, k_{4} T_{4}\right]\right\} \\
& \subset\left(0, Q^{2}\right]
\end{aligned}
$$

and using (47),

$$
\# \bar{U}_{\sigma} \ll \sum_{\substack{1 \leqq k_{4} \leqq K_{4}, 1 \leqq K \leqq L \\ 1 \leqq d \leqq L}} \frac{Q^{2+\eta}}{\left|\frac{T_{4}}{d}\right|^{1-\sigma}}=K_{4} L \frac{Q^{2+\eta}}{T_{4}^{1-\sigma}} \sum_{d \leqq L} d^{1-\sigma} \ll K_{4} L^{3-\sigma} \cdot \frac{Q^{2+\eta}}{T_{4}^{1-\sigma}}
$$

Since the number of divisors of every integer in $\bar{U}_{\sigma}$ is $\ll_{\eta} Q^{\eta}$, the number of triples $\left(a, q_{1}, q_{2}\right)$ with $a q_{1} q_{2} \in \bar{U}_{\sigma}$ fixed is $\ll_{\eta} Q^{3 \eta}$ for $\eta>0$ and therefore

$$
\#\left\{\left(a, q_{1}, q_{2}\right) \in \mathbb{N} \mid a q_{1} q_{2} \in \bar{U}_{\sigma}\right\} \ll_{\eta} Q^{\eta} \cdot \# \bar{U}_{\sigma},
$$

for every fixed $\eta>0$. It follows that

$$
E_{1}^{\prime \prime}=\sum_{1}^{\prime \prime} \ll_{\eta} Q^{\eta} \cdot \# \bar{U}_{\sigma} \cdot \frac{Q^{2}}{\delta_{1} \delta_{2}} \ll_{\eta} K_{4} L^{3-\sigma} \cdot \frac{Q^{4+\eta}}{T_{4}^{1-\sigma} \delta_{1} \delta_{2}} .
$$

Combining this with the result of the second step, for fixed $a$, we have 


$$
\begin{aligned}
\sum_{1}^{\prime}= & \sum_{\substack{q_{1} \leqq Q / \delta_{1}, a q_{1} q_{2} \in U_{\sigma} \\
q_{2} \leqq Q / \delta_{2},\left(q_{1}, q_{2} \delta\right)=1,\left(q_{2}, \delta\right)=1}} \sum=\sum_{\substack{q_{1} \leqq Q / \delta_{1}, q_{2} \leqq Q / \delta_{2},\left(q_{1}, q_{2} \delta\right)=1,\left(q_{2}, \delta\right)=1, a q_{1}, q_{2} \in U_{\sigma}}}\left[\frac{6 Q^{2}}{\pi^{2} \delta_{3} \delta_{4}}\left(\int_{0}^{1} f(x) \mathrm{d} x\right) \prod_{p \mid \delta q_{1} q_{2}}\left(1-\frac{2}{p+1}\right)\right. \\
& \left.\times \iint_{[0,1]^{2}} h\left(q_{1}, q_{2}, \frac{Q}{\delta_{3}} z, \frac{Q}{\delta_{4}} w\right) \mathrm{d} z \mathrm{~d} w+E_{2}\right] .
\end{aligned}
$$

Therefore one has

$$
\begin{aligned}
\sum_{1}=\sum_{\substack{q_{1} \leqq Q / \delta_{1}, q_{2} \leqq Q / \delta_{2},\left(q_{1}, q_{2} \delta\right)=1,\left(q_{2}, \delta\right)=1}}\left[\frac{6 Q^{2}}{\pi^{2} \delta_{3} \delta_{4}}\left(\int_{0}^{1} f(x) \mathrm{d} x\right) \prod_{p \mid \delta q_{1} q_{2}}\left(1-\frac{2}{p+1}\right)\right. \\
\left.\quad \times \iint_{[0,1]^{2}} h\left(q_{1}, q_{2}, \frac{Q}{\delta_{3}} z, \frac{Q}{\delta_{4}} w\right) \mathrm{d} z \mathrm{~d} w+E_{2}\right]+O\left(E_{1}^{\prime \prime}\right) \\
=E_{1}+\frac{6 Q^{2}}{\pi^{2} \delta_{3} \delta_{4}}\left(\int_{0}^{1} f(x) \mathrm{d} x\right) \prod_{p \mid \delta}\left(1-\frac{2}{p+1}\right) \\
\quad \times \int_{\substack{[0,1]^{2} \\
q_{1} \leqq Q / \delta_{1}, p \mid q_{1} q_{2} \\
q_{2} \leqq Q / \delta_{2},\left(q_{1}, q_{2} \delta\right)=1,\left(q_{2}, \delta\right)=1}} \prod\left(1-\frac{2}{p+1}\right) h\left(q_{1}, q_{2}, \frac{Q}{\delta_{3}} z, \frac{Q}{\delta_{4}} w\right) \mathrm{d} z \mathrm{~d} w
\end{aligned}
$$

where

$$
E_{1} \ll E_{1}^{\prime \prime}+Q^{2} E_{2}
$$

In conclusion we have

$$
\begin{aligned}
\sum_{1}= & E_{1}+\frac{6 Q^{2}}{\pi^{2} \delta_{3} \delta_{4}}\left(\int_{0}^{1} f(x) \mathrm{d} x\right) \\
& \times \iint_{[0,1]^{2}} \sum_{\substack{q_{1} \leqq Q / \delta_{1}, p \mid \delta q_{1} q_{2} \\
q_{2} \leqq Q / \delta_{2},\left(q_{1}, q_{2} \delta\right)=1,\left(q_{2}, \delta\right)=1}}\left(1-\frac{2}{p+1}\right) h\left(q_{1}, q_{2}, \frac{Q}{\delta_{3}} z, \frac{Q}{\delta_{4}} w\right) \mathrm{d} z \mathrm{~d} w
\end{aligned}
$$

where $E_{1}$ is estimated above in (61). 
3.5.4. Fourth step. Let us now complete the estimation of the error term $E_{1}$. Note that using $T_{4} K_{4}=Q / \delta_{4}$ in (59), we have

$$
E_{1}^{\prime \prime} \ll_{\eta} \frac{K_{4} L^{3-\sigma} Q^{4+\eta}}{T_{4}^{1-\sigma} \delta_{1} \delta_{2}} \ll_{\eta} K_{4}^{2-\sigma} L^{3-\sigma} Q^{3+\sigma+\eta} .
$$

Also from (58),

$$
E_{2} \ll \frac{Q^{2}}{K_{3}}+\frac{Q^{2}}{K_{4}}+E_{2}^{\prime \prime}
$$

where by (57)

$$
E_{2}^{\prime \prime} \ll K_{3} K_{4} E_{3}+\left(T_{3}+T_{4}\right) Q
$$

Finally combining (54) and (56) one has

$$
E_{3} \ll_{\eta}\left(\frac{T_{3} T_{4}}{L}+Q+Q^{\frac{1}{2}} T_{3}^{\frac{3}{4}} T_{4}^{\frac{3-\sigma}{4}}\right) K^{\prime} Q^{\eta}+\frac{Q^{2}}{\delta_{3} \delta_{4} K_{3} K_{4} K^{\prime}}
$$

Gathering all of the above estimates together,

$$
\begin{aligned}
E_{1} \ll & E_{1}^{\prime \prime}+Q^{2} E_{2} \\
& \ll_{\eta} K_{4}^{2-\sigma} L^{3-\sigma} Q^{3+\sigma+\eta}+\frac{Q^{4}}{K_{3}}+\frac{Q^{4}}{K_{4}}+\frac{Q^{4}}{K^{\prime}} \\
& \quad+Q^{\eta} \cdot\left(Q^{4} \frac{K^{\prime}}{L}+K_{3} K_{4} K^{\prime} Q^{3}+K^{\prime} K_{3}^{\frac{1}{4}} K_{4}^{\frac{1+\sigma}{4}} Q^{4-\frac{\sigma}{4}}\right) .
\end{aligned}
$$

Choosing

$$
L=Q^{\sigma_{1}}, \quad K_{3}=K_{4}=K^{\prime} \approx Q^{\sigma_{2}}
$$

one obtains

$$
\begin{aligned}
E_{1} \ll_{\eta} & Q^{(2-\sigma) \sigma_{2}+(3-\sigma) \sigma_{1}+3+\sigma+\eta}+Q^{4-\sigma_{2}} \\
& +Q^{\eta} \cdot\left(Q^{4+\sigma_{2}-\sigma_{1}}+Q^{3+3 \sigma_{2}}+Q^{\frac{6+\sigma}{4} \sigma_{2}+4-\frac{\sigma}{4}}\right) .
\end{aligned}
$$

Taking $\sigma_{1}=2 \sigma_{2}$, this reduces to

$$
E_{1} \ll_{\eta} Q^{\eta}\left(Q^{3+\sigma\left(1-3 \sigma_{2}\right)+8 \sigma_{2}}+Q^{4-\sigma_{2}}+Q^{3+3 \sigma_{2}}+Q^{\frac{6+\sigma}{4} \sigma_{2}+4-\frac{\sigma}{4}}\right) .
$$

In order to balance all the terms above, it suffices to solve the system

$$
\left\{\begin{array}{l}
3+\sigma\left(1-3 \sigma_{2}\right)+8 \sigma_{2}=4-\sigma_{2} \\
\frac{6+\sigma}{4} \sigma_{2}+4-\frac{\sigma}{4}=4-\sigma_{2}
\end{array}\right.
$$


with $0<\sigma<1$ to obtain that

$$
\sigma_{2}=\frac{10-\sqrt{61}}{39} \approx \frac{1}{17.8}>\frac{1}{18}
$$
For convenience we may take $\sigma_{2}=\frac{1}{18}, \sigma_{1}=\frac{1}{9}, \sigma=\frac{46}{77}$ and arrive at the concluding esti-
mate for $E_{1}$ as

$$
E_{1} \ll_{\eta} Q^{4-\frac{1}{18}+\eta}
$$

3.6. Final reductions. Let

$$
f_{1}(n):=\prod_{p \mid n}\left(1-\frac{2}{p+1}\right)
$$

and note that $f_{1}(n)$ is a multiplicative function with $\left|f_{1}(n)\right| \leqq 1$. Our next objective is to eliminate the dependence on $q_{1}, q_{2}$ in the main term of $\sum_{1}$ from (61). To this end, we consider the sum over $q_{1}, q_{2}$ appearing in $\sum_{1}$ and put

$$
\begin{aligned}
W= & \sum_{\substack{q_{1} \leqq Q / \delta_{1}, q_{2} \leqq Q / \delta_{2},\left(q_{1}, q_{2} \delta\right)=1 \\
\left(q_{2}, \delta\right)=1}} f_{1}\left(\delta q_{1} q_{2}\right) h\left(q_{1}, q_{2}, \frac{Q}{\delta_{3}} z, \frac{Q}{\delta_{4}} w\right) \\
= & \sum_{\substack{1 \leqq q_{1} \leqq Q / \delta_{1},\left(q_{1}, \delta\right)=1}} f_{1}\left(\delta q_{1}\right) \sum_{\substack{q_{2} \leqq Q / \delta_{2},\left(q_{2}, q_{1} \delta\right)=1}} f_{1}\left(q_{2}\right) h\left(q_{1}, q_{2}, \frac{Q}{\delta_{3}} z, \frac{Q}{\delta_{4}} w\right) .
\end{aligned}
$$

If we define the Dirichlet convolution of $\mu$ and $f_{1}$ as

$$
g_{1}(m)=\left(\mu * f_{1}\right)(m)=\sum_{d \mid m} \mu(d) f_{1}\left(\frac{m}{d}\right)
$$

then $f_{1}=1 * g_{1}$ so that for any prime $p$,

$$
g_{1}\left(p^{n}\right)= \begin{cases}-\frac{2}{p+1}, & n=1 \\ 0, & n \geqq 2 .\end{cases}
$$

Therefore one has, $\left|g_{1}(p)\right|<\frac{2}{p}$, and

$$
\left|g_{1}(d)\right| \leqq \frac{2^{\omega(d)}}{d} \quad \text { for every } d \geqq 1,
$$

where $\omega(d)$ is the number of distinct prime divisors of $d$. In this way the inner sum of $W$ becomes 


$$
\begin{aligned}
W_{q_{1}}= & \sum_{\substack{q_{2} \leqq Q / \delta_{2},\left(q_{2}, q_{1} \delta\right)=1}} f_{1}\left(q_{2}\right) h\left(q_{1}, q_{2}, \frac{Q}{\delta_{3}} z, \frac{Q}{\delta_{4}} w\right) \\
= & \sum_{\substack{q_{2} \leqq Q / \delta_{2},\left(q_{2}, q_{1} \delta\right)=1}} h\left(q_{1}, q_{2}, \frac{Q}{\delta_{3}} z, \frac{Q}{\delta_{4}} w\right) \sum_{D \mid q_{2}} g_{1}(D) \\
= & \sum_{\substack{D \leqq Q / \delta_{2},\left(D, q_{1} \delta\right)=1}} g_{1}(D) \sum_{\substack{m \leqq \frac{Q}{D \delta_{2}} \\
\left(m, q_{1} \delta\right)=1}} h\left(q_{1}, m D, \frac{Q}{\delta_{3}} z, \frac{Q}{\delta_{4}} w\right) .
\end{aligned}
$$

For fixed $q_{1}, z, w$ and $D$, define $F(m):=h\left(q_{1}, m D, z, w\right)$, and note that $\|F\|_{\infty} \ll 1$, and from (21),

$$
\left|F^{\prime}(m)\right|=D \cdot\left|\frac{\partial h}{\partial y}\right| \ll \frac{D \delta_{2}}{Q} .
$$

Applying the result (33),

$$
\begin{aligned}
\sum_{\substack{m \leqq \frac{Q}{D \delta_{2}} \\
\left(m, q_{1} \delta\right)=1}} h\left(q_{1}, m D, \frac{Q}{\delta_{3}} z, \frac{Q}{\delta_{4}} w\right) & =\frac{\varphi\left(\delta q_{1}\right)}{\delta q_{1}} \int_{0}^{\frac{Q}{D \delta_{2}}} h\left(q_{1}, D y, \frac{Q}{\delta_{3}} z, \frac{Q}{\delta_{4}} w\right) \mathrm{d} y+E_{F} \\
& =\frac{\varphi\left(\delta q_{1}\right)}{\delta q_{1}} \frac{Q}{D \delta_{2}} \int_{0}^{1} h\left(q_{1}, \frac{Q}{\delta_{2}} y, \frac{Q}{\delta_{3}} z, \frac{Q}{\delta_{4}} w\right) \mathrm{d} y+E_{F}
\end{aligned}
$$

where the error term $E_{F}$ is estimated as

$$
E_{F} \ll_{\eta}\left(\delta q_{1}\right)^{\eta}\left(\frac{D \delta_{2}}{Q} \cdot \frac{Q}{D \delta_{2}}+1\right) \ll_{\eta} Q^{\eta}
$$

Consequently,

$$
\begin{aligned}
W_{q_{1}} & =\sum_{\substack{D \leqq Q / \delta_{2},\left(D, q_{1} \delta\right)=1}} g_{1}(D)\left(\frac{\varphi\left(\delta q_{1}\right)}{\delta q_{1}} \frac{Q}{\delta_{2} D} \int_{0}^{1} h\left(q_{1}, \frac{Q}{\delta_{2}} y, \frac{Q}{\delta_{3}} z, \frac{Q}{\delta_{4}} w\right) \mathrm{d} y+E_{F}\right) \\
& \left.=\frac{Q}{\delta_{2}} \frac{\varphi\left(\delta q_{1}\right)}{\delta q_{1}} \int_{0}^{1} h\left(q_{1}, \frac{Q}{\delta_{2}} y, \frac{Q}{\delta_{3}} z, \frac{Q}{\delta_{4}} w\right) \mathrm{d} y\right)\left(\sum_{\substack{D \leqq Q / \delta_{2},\left(D, q_{1} \delta\right)=1}} \frac{g_{1}(D)}{D}\right)+E_{q_{1}}^{\prime}
\end{aligned}
$$

where the error term $E_{q_{1}}^{\prime}$ is estimated as

$$
E_{q_{1}}^{\prime} \ll \sum_{\substack{D \leqq Q / \delta_{2},\left(D, q_{1} \delta\right)=1}}\left|g_{1}(D)\right| \cdot E_{F} \ll_{\eta}(\log Q)^{2} \cdot Q^{\eta} \ll_{\eta} Q^{\eta},
$$

since $g_{1}(D) \neq 0$ only for square-free $D, 2^{\omega(D)}=\sigma_{0}(D)$ for such $D$ and 


$$
\sum_{D \leqq Q / \delta_{2}}\left|g_{1}(D)\right| \leqq \sum_{D \leqq Q / \delta_{2}} \frac{\sigma_{0}(D)}{D} \ll(\log Q)^{2}
$$

by partial summation. Completing the convergent sum on $D$, we have

$$
\begin{aligned}
\sum_{\substack{D \leqq Q / \delta_{2},\left(D, q_{1} \delta\right)=1}} \frac{g_{1}(D)}{D} & =\sum_{\substack{D \geqq 1,\left(D, q_{1} \delta\right)=1}} \frac{g_{1}(D)}{D}+O\left(\sum_{D>Q / \delta_{2}} \frac{\mu^{2}(D) \sigma_{0}(D)}{D^{2}}\right) \\
& =\frac{\prod_{p}\left(1+\frac{g_{1}(p)}{p}\right)}{\prod_{p \mid \delta q_{1}}\left(1+\frac{g_{1}(p)}{p}\right)}+O\left(\frac{\delta_{2}}{Q^{1-\eta}}\right) \\
& =\frac{\prod_{p}\left(1-\frac{2}{p(p+1)}\right)}{\prod_{p \mid \delta q_{1}}\left(1-\frac{2}{p(p+1)}\right)}+O\left(\frac{\delta_{2}}{Q^{1-\eta}}\right) .
\end{aligned}
$$

Using this we may rewrite $W_{q_{1}}$ as

$$
\left.W_{q_{1}}=\frac{Q}{\delta_{2}} \frac{\varphi\left(\delta q_{1}\right)}{\delta q_{1}} \frac{\prod_{p}\left(1-\frac{2}{p(p+1)}\right)}{\prod_{p \mid \delta q_{1}}\left(1-\frac{2}{p(p+1)}\right)} \int_{0}^{1} h\left(q_{1}, \frac{Q}{\delta_{2}} y, \frac{Q}{\delta_{3}} z, \frac{Q}{\delta_{4}} w\right) \mathrm{d} y\right)+E_{q_{1}}
$$

where the error term $E_{q_{1}}$ is still estimated as

$$
E_{q_{1}} \ll_{\eta} Q^{\eta}
$$

Recall that

Let

$$
W=\sum_{\substack{1 \leqq q_{1} \leqq Q / \delta_{1},\left(q_{1}, \delta\right)=1}} f_{1}\left(\delta q_{1}\right) W_{q_{1}}
$$

$$
f_{2}(n):=\prod_{p \mid n}\left(1-\frac{3}{p+2}\right), \quad n \in \mathbb{N},
$$

and note that $f_{2}$ is a multiplicative function with $\left|f_{2}(n)\right| \leqq 1$. It follows that

$$
\begin{aligned}
f_{1}(n) \frac{\varphi(n)}{n} \frac{1}{\prod_{p \mid n}\left(1-\frac{2}{p(p+1)}\right)} & =\prod_{p \mid n} \frac{\left(1-\frac{2}{p+1}\right)\left(1-\frac{1}{p}\right)}{\left(1-\frac{2}{p(p+1)}\right)} \\
& =\prod_{p \mid n}\left(1-\frac{3}{p+2}\right)=f_{2}(n),
\end{aligned}
$$


and rewriting $W$ gives

$$
W=\frac{c_{1} Q}{\delta_{2}} \int_{0}^{1} \sum_{\substack{1 \leqq q_{1} \leqq Q / \delta_{1},\left(q_{1}, \delta\right)=1}} f_{2}\left(\delta q_{1}\right) h\left(q_{1}, \frac{Q}{\delta_{2}} y, \frac{Q}{\delta_{3}} z, \frac{Q}{\delta_{4}} w\right) \mathrm{d} y+E_{W}^{\prime}
$$

where

$$
c_{1}=\prod_{p \text { prime }}\left(1-\frac{2}{p(p+1)}\right)>0
$$

and the error term can be estimated as

$$
E_{W}^{\prime} \ll \frac{Q}{\delta_{1}} \cdot E_{q_{1}} \ll_{\eta} Q^{1+\eta}
$$

Similarly, if we define $g_{2}:=\mu * f_{2}$, then $f_{2}=1 * g_{2}$ and for any prime $p$,

$$
g_{2}\left(p^{n}\right)= \begin{cases}-\frac{3}{p+2}, & n=1, \\ 0, & n \geqq 2\end{cases}
$$

so that for every $d \geqq 1$,

$$
\left|g_{2}(d)\right| \leqq \frac{3^{\omega(d)}}{d} \leqq \frac{2^{2 \omega(d)}}{d}
$$

We may now rewrite the inner sum inside the integral for $W$ as

$$
\begin{aligned}
W_{1} & =\sum_{\substack{q_{1} \leqq Q / \delta_{1},\left(q_{1}, \delta\right)=1}} f_{2}(\delta) f_{2}\left(q_{1}\right) h\left(q_{1}, \frac{Q}{\delta_{2}} y, \frac{Q}{\delta_{3}} z, \frac{Q}{\delta_{4}} w\right) \\
& =f_{2}(\delta) \sum_{\substack{q_{1} \leqq Q / \delta_{1},\left(q_{1}, \delta\right)=1}} h\left(q_{1}, \frac{Q}{\delta_{2}} y, \frac{Q}{\delta_{3}} z, \frac{Q}{\delta_{4}} w\right) \sum_{D \mid q_{1}} g_{2}(D) \\
& =f_{2}(\delta) \sum_{\substack{D \leqq Q / \delta_{1},(D, \delta)=1}} g_{2}(D) \sum_{\substack{m \leqq \frac{Q}{D \delta_{1}} \\
(m, \delta)=1}} h\left(m D, \frac{Q}{\delta_{2}} y, \frac{Q}{\delta_{3}} z, \frac{Q}{\delta_{4}} w\right) .
\end{aligned}
$$

Using (33) and (20), we obtain

$$
\sum_{\substack{m \leqq \frac{Q}{D \delta_{1}},(m, \delta)=1}} h\left(m D, \frac{Q}{\delta_{2}} y, \frac{Q}{\delta_{3}} z, \frac{Q}{\delta_{4}} w\right)=\frac{\varphi(\delta)}{\delta} \frac{Q}{D \delta_{1}} \times \int_{0}^{1} h\left(\frac{Q}{\delta_{1}} x, \frac{Q}{\delta_{2}} y, \frac{Q}{\delta_{3}} z, \frac{Q}{\delta_{4}} w\right) \mathrm{d} y+O(1)
$$

and completing the convergent sum on $D$, 


$$
\begin{aligned}
\sum_{\substack{D \leqq Q / \delta_{1},(D, \delta)=1}} \frac{g_{2}(D)}{D}= & \sum_{\substack{D \geqq 1,(D, \delta)=1}} \frac{g_{2}(D)}{D}+O\left(\sum_{D>Q / \delta_{1}} \frac{\mu^{2}(D) \sigma_{0}^{2}(D)}{D^{2}}\right) \\
& =\frac{\prod_{p}\left(1+\frac{g_{2}(p)}{p}\right)}{\prod_{p \mid \delta}\left(1+\frac{g_{2}(p)}{p}\right)}+O\left(\frac{\delta_{1}}{Q^{1-\eta}}\right) \\
& =\frac{\prod_{p}\left(1-\frac{3}{p(p+2)}\right)}{\prod_{p \mid \delta}\left(1-\frac{3}{p(p+2)}\right)}+O\left(\frac{\delta_{1}}{Q^{1-\eta}}\right) .
\end{aligned}
$$

Moreover using

$$
\left|g_{2}(D)\right| \leqq \frac{\sigma_{0}^{2}(D)}{D}
$$

and the well-known estimate

$$
\sum_{m \leqq t} \sigma_{0}^{2}(m) \ll t(\log t)^{3},
$$

we obtain by partial summation that

$$
\sum_{\substack{D \leqq Q / \delta_{1} \\(D, \delta)=1}}\left|g_{2}(D)\right| \ll(\log Q)^{4} \ll_{\eta} Q^{\eta}
$$

In conclusion we have

$$
W_{1}=f_{2}(\delta) \frac{Q}{\delta_{1}} \frac{\varphi(\delta)}{\delta} \frac{\prod_{p}\left(1-\frac{3}{p(p+2)}\right)}{\prod_{p \mid \delta}\left(1-\frac{3}{p(p+2)}\right)} \int_{0}^{1} h\left(\frac{Q}{\delta_{1}} x, \frac{Q}{\delta_{2}} y, \frac{Q}{\delta_{3}} z, \frac{Q}{\delta_{4}} w\right) \mathrm{d} x+E^{*}
$$

where the error term $E^{*}$ is estimated as

$$
E^{*} \ll_{\eta} Q^{\eta}
$$

This completes the estimation of all error terms. Denoting

$$
c_{2}=\prod_{p \text { prime }}\left(1-\frac{3}{p(p+2)}\right)>0,
$$

and the multiplicative function

$$
c(n)=f_{2}(n) \frac{\varphi(n)}{n} \frac{1}{\prod_{p \mid n}\left(1-\frac{3}{p(p+2)}\right)}=\prod_{p \mid n}\left(1-\frac{4}{p+3}\right)
$$


we rewrite $\sum_{1}$ from $(61)$ as

$$
\begin{aligned}
\sum_{1}= & \frac{6 c_{1} c_{2} c(\delta) Q^{4}}{\pi^{2} \delta_{1} \delta_{2} \delta_{3} \delta_{4}} \cdot\left(\int_{0}^{1} f(x) \mathrm{d} x\right) \\
& \times \iint_{[0,1]^{4}} h\left(\frac{Q}{\delta_{1}} x, \frac{Q}{\delta_{2}} y, \frac{Q}{\delta_{3}} z, \frac{Q}{\delta_{4}} w\right) \mathrm{d} x \mathrm{~d} y \mathrm{~d} z \mathrm{~d} w+E .
\end{aligned}
$$

Since $E_{1} \ll_{\eta} Q^{4-\frac{1}{18}+\eta}, Q^{2} E_{W}^{\prime} \ll_{\eta} Q^{3+\eta}$ and $Q^{3} E^{*} \ll_{\eta} Q^{3+\eta}$, the final error term $E$ can be estimated as

$$
E \ll E_{1}+Q^{2} E_{W}^{\prime}+Q^{3} E^{*} \ll_{\eta} Q^{4-\frac{1}{18}+\eta}+Q^{3+\eta} \ll_{\eta} Q^{4-\frac{1}{18}+\eta},
$$

for $0<\eta<\frac{1}{18}$.

Using (18) we have

$$
h\left(\frac{Q}{\delta_{1}} x, \frac{Q}{\delta_{2}} y, \frac{Q}{\delta_{3}} z, \frac{Q}{\delta_{4}} w\right) \neq 0 \Rightarrow c_{\wedge} \leqq x, y, z, w \leqq 1,
$$

and combining (11), (12) and (14),

$$
h\left(\frac{Q}{\delta_{1}} x, \frac{Q}{\delta_{2}} y, \frac{Q}{\delta_{3}} z, \frac{Q}{\delta_{4}} w\right)=H\left(\frac{N m \delta r_{1} r_{2} r_{3} r_{4}}{Q^{4} x y z w}\right) .
$$

By (2), we have

$$
\frac{N}{Q^{4}}=\frac{9}{\pi^{4}}+O_{\eta}\left(Q^{-1+\eta}\right)
$$

so that

$$
\left|H\left(\frac{N m \delta r_{1} r_{2} r_{3} r_{4}}{Q^{4} x y z w}\right)-H\left(\frac{9 m \delta r_{1} r_{2} r_{3} r_{4}}{\pi^{4} x y z w}\right)\right| \ll_{\eta} Q^{-1+\eta} .
$$

Letting $c=c_{1} c_{2}$ and rewriting $\sum_{1}$, we get

$$
\begin{aligned}
\sum_{1}= & \frac{6 c c(\delta) Q^{4}}{\pi^{2} \delta_{1} \delta_{2} \delta_{3} \delta_{4}} \cdot\left(\int_{0}^{1} f(x) \mathrm{d} x\right) \\
& \times \iint_{[0,1]^{4}} H\left(\frac{9 m \delta r_{1} r_{2} r_{3} r_{4}}{\pi^{4} x y z w}\right) \mathrm{d} x \mathrm{~d} y \mathrm{~d} z \mathrm{~d} w+E,
\end{aligned}
$$

where

$$
E \ll_{\eta} Q^{4-\frac{1}{18}+\eta}
$$


Recall that, by (11),

$$
\begin{aligned}
\int_{0}^{1} f(x) \mathrm{d} x & =\int_{0}^{1} G\left(\frac{m x+n}{\tilde{u}}\right)^{2} \mathrm{~d} x=\frac{\tilde{u}}{m} \int_{\frac{n}{\tilde{u}}}^{\frac{m+n}{\tilde{u}}} G(z)^{2} \mathrm{~d} z \\
& =\frac{\tilde{u}}{m}\left(\int_{\frac{n}{\tilde{u}}}^{\frac{n+1}{\tilde{u}}} G(z)^{2} \mathrm{~d} z+\cdots+\int_{\frac{n+m-1}{\tilde{u}}}^{\frac{m+n}{\tilde{u}}} G(z)^{2} \mathrm{~d} z\right),
\end{aligned}
$$

and

$$
\sum_{n \in \mathbb{Z}} \int_{0}^{1} f(x) \mathrm{d} x=\frac{\tilde{u}}{m} m \cdot \int_{0}^{1} G(z)^{2} \mathrm{~d} x=\tilde{u} \int_{0}^{1} G(z)^{2} \mathrm{~d} x .
$$

Recall that $\mathcal{A}$ is the finite set of all integers $n$ satisfying

$$
0<\frac{\widetilde{a_{2}} m}{\left[d_{1}, d_{2}\right]}+\frac{n}{\tilde{u}}<1
$$

Summing over all $n \in \mathcal{A}$, we obtain from (8) that

$$
\begin{aligned}
& \sum_{a_{i} \leqq Q / r_{i}, 1 \leqq i \leqq 4} \frac{1}{\left(\left[a_{1}, a_{2}\right],\left[a_{3}, a_{4}\right]\right)} \sum_{n \in \mathcal{A}} \sum_{1} \\
& a_{1}, a_{2}, a_{3}, a_{4} \in P_{\delta} \text {, } \\
& \frac{a_{1} a_{2} a_{3} a_{4}}{\left[a_{1}, a_{2}, a_{3}, a_{4}\right]}=\delta \\
& =\sum_{\substack{a_{i} \leqq Q / r_{i}, 1 \leqq i \leq 4, a_{1}, a_{2}, a_{3}, a_{4} \in \bar{P}_{\delta}, a_{1} a_{2} a_{4} a_{4} \\
\left(a_{1}, a_{2}, a_{3}, a_{4}=\delta,\left(\left[a_{1}, a_{2}\right],\left[a_{3}, a_{4}\right]\right)=\tilde{u}\right.}} \frac{1}{\tilde{u}}\left\{\frac{6 c c(\delta) Q^{4}}{\pi^{2} \delta_{1} \delta_{2} \delta_{3} \delta_{4}} \tilde{u}\left(\int_{0}^{1} G(z)^{2} \mathrm{~d} z\right)\right. \\
& \left.\times \iiint_{[0,1]^{4}} H\left(\frac{9 m \delta r_{1} r_{2} r_{3} r_{4}}{\pi^{4} x y z w}\right) \mathrm{d} x \mathrm{~d} y \mathrm{~d} z \mathrm{~d} w+E\right\}
\end{aligned}
$$

where $\sum_{1}$ is defined as in (9). If $\delta=1$, then $P_{\delta}=\{1\}$ and $a_{1}=a_{2}=a_{3}=a_{4}=1$. Otherwise $\delta \geqq 2$, and we consider the prime factorization of $\delta$ as

$$
\delta=p_{1}^{e_{1}} p_{2}^{e_{2}} \cdots p_{k}^{e_{k}}, \quad e_{1}, \ldots, e_{k} \geqq 1 .
$$

Writing the prime factorizations of $a_{i}$ as

$$
a_{i}=p_{1}^{e_{i 1}} p_{2}^{e_{i 2}} \cdots p_{k}^{e_{i k}}, \quad 1 \leqq i \leqq 4,
$$

the condition $\frac{a_{1} a_{2} a_{3} a_{4}}{\left[a_{1}, a_{2}, a_{3}, a_{4}\right]}=\delta$ implies that

$$
e_{1 j}+e_{2 j}+e_{3 j}+e_{4 j}-\max \left\{e_{1 j}, e_{2 j}, e_{3 j}, e_{4 j}\right\}=e_{j},
$$


for $1 \leqq j \leqq k$. Since $a_{i} \leqq Q / r_{i} \leqq Q$, it follows that

$$
e_{i j} \log 2 \leqq e_{i 1} \log p_{1}+\cdots+e_{i k} \log p_{k} \leqq \log Q-\log r_{i}
$$

and

$$
0 \leqq e_{i j} \leqq \frac{\log Q}{\log 2}
$$

for any $1 \leqq i \leqq 4$ and $1 \leqq j \leqq k$. Since $\delta$ is absolutely bounded and $\omega(\delta)=k, k$ is absolutely bounded and

$$
\sum_{\substack{\left.a_{i} \leqq Q / r_{i}, 1 \leqq i \leqq 4 \\ a_{1}, a_{2}, a_{3}, a_{4} \in \bar{P}_{\delta}, \frac{1}{a_{1} a_{2} a_{3} a_{4}}, a_{2}, a_{3}, a_{4}\right]=\delta}} 1 \leqq\left(\frac{\log Q}{\log 2}\right)^{4 k} \ll(\log Q)^{4 k} \ll_{\eta} Q^{\eta} .
$$

Since $\delta_{i}=a_{i} r_{i}, 1 \leqq i \leqq 4$, we also have from $(65)$

$$
\begin{aligned}
& \sum_{\substack{a_{i} \leqq Q / r_{i}, 1 \leqq i \leqq 4 \\
a_{1}, a_{2}, a_{3}, a_{1} \in \bar{P}_{\delta}, \frac{a_{1} a_{2} a_{3} a_{4}}{\left[a_{1}, a_{2}, a_{3}, a_{4}\right]}=\delta}} \frac{6 c c(\delta) Q^{4}}{\pi^{2} \delta_{1} \delta_{2} \delta_{3} \delta_{4}}\left(\int_{0}^{1} G(z)^{2} \mathrm{~d} z\right) \\
& \times \iiint_{[0,1]^{4}} H\left(\frac{9 m \delta r_{1} r_{2} r_{3} r_{4}}{\pi^{4} x y z w}\right) \mathrm{d} x \mathrm{~d} y \mathrm{~d} z \mathrm{~d} w+E \\
& =\frac{6 c c(\delta)}{\pi^{2}}\left(\int_{0}^{1} G(z)^{2} \mathrm{~d} z\right) \iint_{[0,1]^{4}} H\left(\frac{9 m \delta r_{1} r_{2} r_{3} r_{4}}{\pi^{4} x y z w}\right) \mathrm{d} x \mathrm{~d} y \mathrm{~d} z \mathrm{~d} w \\
& \times \frac{Q^{4}}{r_{1} r_{2} r_{3} r_{4}} \sum_{\substack{a_{i} \leqq Q / r_{i}, 1 \leqq i \leqq 4 \\
a_{1}, a_{2}, a_{3}, a_{4} \in \bar{P}_{\delta}, a_{1} a_{2} a_{3} a_{4} \\
\left[a_{1}, a_{2}, a_{3}, a_{4}\right]}} \frac{1}{a_{1} a_{2} a_{3} a_{4}}+E,
\end{aligned}
$$

where, by (64)

$$
E \ll_{\eta} Q^{4-\frac{1}{18}+\eta}
$$

Our next goal is to estimate the sum

$$
A_{\delta, r_{1}, r_{2}, r_{3}, r_{4}}(Q)=\sum_{\substack{a_{i} \leqq Q / r_{i}, 1 \leqq i \leqq 4, a_{1}, a_{2}, a_{3}, a_{4} \in \bar{P}_{\delta} \\ \frac{a_{1} a_{2} a_{3} a_{4}}{\left[a_{1}, a_{2}, a_{3}, a_{4}\right]}=\delta}} \frac{1}{a_{1} a_{2} a_{3} a_{4}} .
$$

Again we may assume $\delta \geqq 2$ and (67), (68), (69). Since $A_{\delta, r_{1}, r_{2}, r_{3}, r_{4}}(Q)$ is increasing as $Q \rightarrow \infty$, we deduce that 


$$
\begin{aligned}
A_{\delta, r_{1}, r_{2}, r_{3}, r_{4}}(Q) & \leqq \sum_{e_{i j}} \frac{1}{p_{1}^{e_{11}+e_{21}+e_{31}+e_{41}} p_{2}^{e_{12}+e_{22}+e_{32}+e_{42}} \cdots p_{k}^{e_{1 k}+e_{2 k}+e_{3 k}+e_{4 k}}} \\
& =\left(\sum_{e=0}^{\infty} \frac{1}{p_{1}^{e}}\right)^{4} \cdots\left(\sum_{e=0}^{\infty} \frac{1}{p_{k}^{e}}\right)^{4} \\
& =\left(\prod_{j=1}^{k} \frac{1}{1-p_{j}^{-1}}\right)^{4}<\infty
\end{aligned}
$$

and consequently

$$
\lim _{Q \rightarrow \infty} A_{\delta, r_{1}, r_{2}, r_{3}, r_{4}}(Q)=A(\delta)
$$

for some constant $A(\delta)$ depending only on $\delta$. Denoting the condition (69) as $\left(e_{i j}\right) \in P P$, we obtain

$$
A\left(p^{m}\right)=\sum_{\substack{e_{i j} \geqq 0,\left(e_{i j}\right) \in P P}} \frac{1}{p_{1}^{e_{11}+e_{21}+e_{31}+e_{41}} p_{2}^{e_{12}+e_{22}+e_{32}+e_{42}} \cdots p_{k}^{e_{1 k}+e_{2 k}+e_{3 k}+e_{4 k}}} .
$$

It is easy to see that $A$ is a multiplicative function and for any prime number $p$ and integer $m \geqq 1$,

$$
A\left(p^{m}\right)=\sum_{\substack{e_{1}, e_{2}, e_{3}, e_{4} \geqq 0, e_{1}+e_{2}+e_{3}+e_{4}-\max \left\{e_{1}+e_{2}+e_{3}+e_{4}\right\}=m}} \frac{1}{p^{e_{1}+e_{2}+e_{3}+e_{4}}} .
$$

Furthermore,

$$
\begin{aligned}
\left|A_{\delta, r_{1}, r_{2}, r_{3}, r_{4}}(Q)-A(\delta)\right|< & \sum_{e_{11} \log p_{1}+\cdots+e_{1 k} \log p_{k}>\log Q-\log r_{1}} \cdots \\
& +\sum_{e_{21} \log p_{1}+\cdots+e_{2 k} \log p_{k}>\log Q-\log r_{2}} \cdots \\
& +\sum_{e_{31} \log p_{1}+\cdots+e_{3 k} \log p_{k}>\log Q-\log r_{3}} \cdots \\
& +\sum_{e_{41} \log p_{1}+\cdots+e_{4 k} \log p_{k}>\log Q-\log r_{4}} \cdots \\
= & \Omega_{1}+\Omega_{2}+\Omega_{3}+\Omega_{4},
\end{aligned}
$$

where

$$
\Omega_{1}=\left(\prod_{j=1}^{k} \frac{1}{1-p_{j}^{-1}}\right)^{3} \Omega_{1}^{\prime}
$$

and

$$
\Omega_{1}^{\prime}=\sum_{e_{11} \log p_{1}+\cdots+e_{1 k} \log p_{k}>\log Q-\log r_{1}} \frac{1}{p_{1}^{e_{11}} \cdots p_{k}^{e_{1 k}}}
$$


We can estimate $\Omega_{1}^{\prime}$ as

$$
\begin{aligned}
& \Omega_{1}^{\prime}<\sum_{e_{11} \log p_{1}>\log Q-\log r_{1}} \frac{1}{p_{1}^{e_{11}} \cdots p_{k}^{e_{1 k}}}+\cdots+\sum_{e_{1 k} \log p_{k}>\log Q-\log r_{1}} \frac{1}{p_{1}^{e_{11}} \cdots p_{k}^{e_{1 k}}}
\end{aligned}
$$

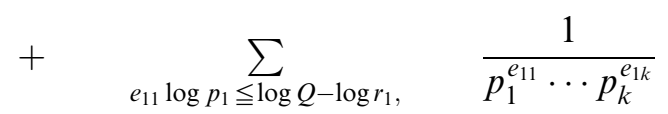

$$
\begin{aligned}
& e_{1 k} \log p_{k} \leqq \log Q-\log r_{1}, \\
& e_{11} \log p_{1}+\cdots+e_{1 k} \log p_{k}>\log Q-\log r_{1} \\
& =\Omega_{1,1}+\cdots+\Omega_{1, k}+\Omega_{1}^{\prime \prime} \text {. }
\end{aligned}
$$

Note that for $\Omega_{1,1}, e_{12}, \ldots, e_{1 k}$ run through all nonnegative integers and since

$$
\frac{1}{p_{1}^{e_{11}}}<\frac{r_{1}}{Q}
$$

it follows that

$$
\begin{aligned}
\Omega_{1,1} & =\left(\prod_{j=2}^{k} \frac{1}{1-p_{j}^{-1}}\right)_{e_{11} \log p_{1}>\log Q-\log r_{1}} \frac{1}{p_{1}^{e_{11}}} \\
& \leqq\left(\prod_{j=2}^{k} \frac{1}{1-p_{j}^{-1}}\right) \frac{\frac{r_{1}}{Q}}{1-p_{1}^{-1}} \ll \frac{1}{Q} .
\end{aligned}
$$

Similarly,

$$
\Omega_{1, j} \ll \frac{1}{Q}, \quad \text { for } 1 \leqq j \leqq k .
$$

On the other hand, $\Omega_{1}^{\prime \prime}$ can be estimated as

$$
\begin{aligned}
\Omega_{1}^{\prime \prime} & \sum_{e_{11} \log p_{1} \leqq \log Q-\log r_{1},} \frac{r_{1}}{\vdots} \\
& e_{1 k} \log p_{k} \leqq \log Q-\log r_{1} \\
& \leqq \\
& \frac{r_{1}}{Q}\left(\frac{\log Q-\log r_{1}}{\log p_{1}}+1\right) \cdots\left(\frac{\log Q-\log r_{1}}{\log p_{k}}+1\right) \\
& \ll \frac{(\log Q)^{k}}{Q} \ll_{\eta} Q^{-1+\eta} .
\end{aligned}
$$

Combining all these estimates finally gives

$$
\Omega_{1} \ll_{\eta} Q^{-1+\eta}
$$

Similar estimates hold true for $\Omega_{2}, \Omega_{3}$ and $\Omega_{4}$, and we have 


$$
A_{\delta, r_{1}, \ldots, r_{4}}(Q)=A(\delta)+O_{\eta}\left(Q^{-1+\eta}\right) .
$$

Returning to $\sum$ from (5) and also to $S_{Q, \mathbf{I}, H, G}$, we have in conclusion that

$$
\begin{aligned}
S_{Q, \mathbf{I}, H, G}= & \frac{6 c Q^{4}}{\pi^{2}}\left(\int_{0}^{1} G(z)^{2} \mathrm{~d} z\right)_{m r_{1} r_{2} r_{3} r_{4} \delta \leqq C_{\curlywedge}} \frac{\mu\left(r_{1}\right) \mu\left(r_{2}\right) \mu\left(r_{3}\right) \mu\left(r_{4}\right)}{r_{1} r_{2} r_{3} r_{4}} \\
& \times c(\delta) A(\delta) \delta \iint_{[0,1]^{4}} H\left(\frac{9 m \delta r_{1} r_{2} r_{3} r_{4}}{\pi^{4} x y z w}\right) \mathrm{d} x \mathrm{~d} y \mathrm{~d} z \mathrm{~d} w+E,
\end{aligned}
$$

where, by (64)

$$
E \ll_{\eta} Q^{4-\frac{1}{18}+\eta}+Q^{3+\eta} \ll_{\eta} Q^{4-\frac{1}{18}+\eta}
$$

for any $0<\eta<\frac{1}{18}$.

3.7. Completion of the proof. To complete the proof of Lemma 3 and to arrive at the simple formula promised for the pair correlation function in Theorem 1, we need to do further calculations. Recall that $C_{\wedge}=\frac{\pi^{4} \wedge}{9}$ and write $S_{Q, \mathbf{I}, H, G}$ as

$$
S_{Q, \mathbf{I}, H, G}=\frac{6 c Q^{4}}{\pi^{2}}\left(\int_{0}^{1} G(z)^{2} \mathrm{~d} z\right) S_{\wedge}+E
$$

where

$$
S_{\wedge}=\sum_{1 \leqq k \leqq \frac{\pi^{4} \wedge}{9}} \frac{H_{k}}{k} \sum_{m r_{1} r_{2} r_{3} r_{4} \delta=k} \frac{k \mu\left(r_{1}\right) \mu\left(r_{2}\right) \mu\left(r_{3}\right) \mu\left(r_{4}\right)}{r_{1} r_{2} r_{3} r_{4}} c(\delta) A(\delta) \delta,
$$

and

$$
H_{k}=\iint_{[0,1]^{4}} H\left(\frac{9 k}{\pi^{4} x y z w}\right) \mathrm{d} x \mathrm{~d} y \mathrm{~d} z \mathrm{~d} w
$$

First, for fixed $k$ with $1 \leqq k \leqq C_{\wedge}$, let

$$
\Omega=[0,1]^{4} \cap\left\{(x, y, z, w) \in \mathbb{R}^{4} \mid x y z w>\frac{9 k}{\pi^{4} \wedge}\right\} .
$$

Changing variables by $x^{\prime}=\frac{9 k}{\pi^{4} x y z w}, y^{\prime}=y, z^{\prime}=z, w^{\prime}=w, \Omega$ is mapped to the region

$$
\Omega^{\prime}=\left\{\left(x^{\prime}, y^{\prime}, z^{\prime}, w^{\prime}\right) \in \mathbb{R}^{4} \mid 0 \leqq y^{\prime}, z^{\prime}, w^{\prime} \leqq 1, \frac{9 k}{\pi^{4} y^{\prime} z^{\prime} w^{\prime}} \leqq x^{\prime}<\wedge\right\}
$$

Using the Jacobian of the transformation 


$$
\left|\frac{\partial(x, y, z, w)}{\partial\left(x^{\prime}, y^{\prime}, z^{\prime}, w^{\prime}\right)}\right|=\frac{9 k}{\pi^{4} x^{\prime 2} y^{\prime} z^{\prime} w^{\prime}},
$$

we have

$$
H_{k}=\iint_{\Omega} H\left(\frac{9 k}{\pi^{4} x y z w}\right) \mathrm{d} x \mathrm{~d} y \mathrm{~d} z \mathrm{~d} w=\iint_{\Omega^{\prime}} H\left(x^{\prime}\right) \frac{9 k / \pi^{4}}{y^{\prime} z^{\prime} w^{\prime}} \frac{1}{x^{\prime 2}} \mathrm{~d} x^{\prime} \mathrm{d} y^{\prime} \mathrm{d} z^{\prime} \mathrm{d} w^{\prime} .
$$

Changing the dummy variables $x^{\prime}, y^{\prime}, z^{\prime}, w^{\prime}$ back to $x, y, z, w$, this further gives

$$
H_{k}=\frac{9 k}{\pi^{4}} \int_{\frac{9 k}{\pi^{4}}}^{\wedge} \frac{H(x)}{x^{2}} U\left(\frac{9 k}{\pi^{4} x}\right) \mathrm{d} x
$$

where the function $U:(0,1] \rightarrow[0, \infty)$ is given by

$$
\begin{aligned}
U(t) & =\int_{t}^{1} \int_{\frac{t}{y}}^{1} \int_{\frac{t}{y z}}^{1} \frac{\mathrm{d} w}{w} \frac{\mathrm{d} z}{z} \frac{\mathrm{d} y}{y}=\int_{t}^{1} \int_{\frac{t}{y}}^{1}\left(\log z+\log \frac{y}{t}\right) \frac{\mathrm{d} z}{z} \frac{\mathrm{d} y}{y} \\
& =\int_{t}^{1} \frac{1}{2} \log ^{2}\left(\frac{y}{t}\right) \frac{\mathrm{d} y}{y}=\frac{1}{6} \log ^{3}\left(\frac{1}{t}\right) .
\end{aligned}
$$

Thus

$$
H_{k}=\frac{3 k}{2 \pi^{4}} \int_{\frac{9 k}{\pi^{4}}}^{\wedge} \frac{H(x)}{x^{2}} \log ^{3} \frac{\pi^{4} x}{9 k} \mathrm{~d} x
$$

Next, we define a multiplicative function $B$ by letting $B(n)=c(n) A(n) n^{2}$ for any $n \in \mathbb{N}$ and a multiplicative function $\psi$ by the convolution

$$
\psi=\mu * \mu * \mu * \mu * I_{d} * B
$$

where $I_{d}$ is the identity function. It is easy to see that

$$
\begin{aligned}
& \sum_{m r_{1} r_{2} r_{3} r_{4} \delta=k} \frac{k \mu\left(r_{1}\right) \mu\left(r_{2}\right) \mu\left(r_{3}\right) \mu\left(r_{4}\right)}{r_{1} r_{2} r_{3} r_{4}} c(\delta) A(\delta) \delta \\
& =\sum_{m r_{1} r_{2} r_{3} r_{4} \delta=k} m \mu\left(r_{1}\right) \mu\left(r_{2}\right) \mu\left(r_{3}\right) \mu\left(r_{4}\right) c(\delta) A(\delta) \delta^{2} \\
& =\mu * \mu * \mu * \mu * I_{d} * B=\psi(k) .
\end{aligned}
$$

Returning to $S_{\wedge}$ from $(72)$, we get

$$
\begin{aligned}
S_{\wedge} & =\sum_{1 \leqq k \leqq \frac{\pi^{4}}{9}} \frac{3 \psi(k)}{2 \pi^{4}}\left(\int_{\frac{9 k}{\pi^{4}}}^{\wedge} \frac{H(x)}{x^{2}} \log ^{3} \frac{\pi^{4} x}{9 k} \mathrm{~d} x\right) \\
& =\frac{3}{2 \pi^{4}}\left(\int_{0}^{\wedge} \frac{H(x)}{x^{2}} \sum_{1 \leqq k \leqq \frac{\pi^{4} \wedge}{9}} \psi(k) \max \left\{0, \log ^{3} \frac{\pi^{4} x}{9 k}\right\} \mathrm{d} x\right) \\
& =\frac{3}{2 \pi^{4}}\left(\int_{0}^{\wedge} \frac{H(x)}{x^{2}} \sum_{1 \leqq k \leqq \frac{\pi^{4} x}{9}} \psi(k) \log ^{3} \frac{\pi^{4} x}{9 k} \mathrm{~d} x\right) .
\end{aligned}
$$


From (72) we finally obtain

$$
S_{Q, \mathbf{I}, H, G}=\frac{9 Q^{4}}{\pi^{4}}\left(\int_{0}^{1} G(z)^{2} \mathrm{~d} z\right)\left(\int_{0}^{\wedge} \frac{H(x)}{x^{2}} \sum_{1 \leqq k \leqq \frac{\pi^{4} x}{9}} \frac{c \psi(k)}{\pi^{2}} \log ^{3} \frac{\pi^{4} x}{9 k} \mathrm{~d} x\right)+E
$$

where

$$
E \ll_{\eta} Q^{4-\frac{1}{18}+\eta}
$$

Our last step is to compute the Zeta-function for $\psi$ explicitly. For any $k, m \geqq 1$, define a function $H$ by

$$
H(k, m)=\sum_{\substack{0 \leqq n_{1}, n_{2}, n_{3}, n_{4} \leqq k \\ n_{1}+n_{2}+n_{3}+n_{4}=m}} 1 .
$$

By the definition of $A$ from (71), we see that for any prime $p$ and integer $m \geqq 1$,

$$
\begin{aligned}
& A\left(p^{m}\right)=\sum_{\substack{e_{1}, e_{2}, e_{3}, e_{4} \geqq 0, e_{1}+e_{2}+e_{3}+e_{4}-\max \left\{e_{1}, e_{2}, e_{3}, e_{4}\right\}=m}} \frac{1}{p^{e_{1}+e_{2}+e_{3}+e_{4}}} \\
& =\sum_{k=1}^{\infty} \frac{1}{p^{k+m}} \sum_{\substack{e_{1}+e_{2}+e_{3}+e_{4}=k+m \\
\max \left\{e_{1}, e_{2}, e_{3}, e_{4}\right\}=k}} 1 \\
& =\sum_{k=1}^{\infty} \frac{1}{p^{k+m}}\left(\sum_{\substack{0 \leqq e_{1}, e_{2}, e_{3}, e_{4} \leqq k, e_{1}+e_{2}+e_{3}+e_{4}=k+m}} 1-\sum_{\substack{0 \leqq e_{1}, e_{2}, e_{3}, e_{4} \leqq k-1, e_{1}+e_{2}+e_{3}+e_{4}=k+m}} 1\right) \\
& =\sum_{k=1}^{\infty} \frac{H(k, k+m)-H(k-1, k+m)}{p^{k+m}} .
\end{aligned}
$$

Then for $s \in \mathbb{C}$ with $\operatorname{Re}(s)$ sufficiently large and for any prime number $p$,

$$
\begin{aligned}
H_{p}(s) & =1+\sum_{m=1}^{\infty} \frac{B\left(p^{m}\right)}{p^{m s}}=1+\sum_{m=1}^{\infty} \frac{p^{2 m}\left(1-\frac{4}{p(p+3)}\right) A\left(p^{m}\right)}{p^{m s}} \\
& =1+\sum_{k=1}^{\infty} \sum_{m=1}^{\infty} \frac{p^{2 m}\left(1-\frac{4}{p(p+3)}\right)(H(k, k+m)-H(k-1, k+m))}{p^{m s} p^{k+m}} \\
& =1+\left(1-\frac{4}{p(p+3)}\right) \sum_{k=1}^{\infty} p^{k(s-2)} \sum_{m=k+1}^{\infty}\left(\frac{H(k, m)}{p^{m(s-1)}}-\frac{H(k-1, m)}{p^{m(s-1)}}\right) .
\end{aligned}
$$

It is easy to see that

$$
\sum_{m=0}^{\infty} H(k, m) q^{m}=\sum_{0 \leqq n_{1}, n_{2}, n_{3}, n_{4} \leqq k} q^{n_{1}+n_{2}+n_{3}+n_{4}}=\left(\frac{1-q^{k+1}}{1-q}\right)^{4}
$$


for $0 \leqq m \leqq k$,

$$
H(k, m)=\sum_{\substack{n_{1}, n_{2}, n_{3}, n_{4} \geq 0, n_{1}+n_{2}+n_{3}+n_{4}=m}} 1=\left(\begin{array}{c}
m+3 \\
3
\end{array}\right)
$$

and for $k \geqq 1$,

$$
H(k, k)-H(k-1, k)=\sum_{\substack{0 \leqq n_{1}, n_{2}, n_{3}, n_{4} \leqq k, n_{1}+n_{2}+n_{3}+n_{4}=k, \max \left\{n_{1}, n_{2}, n_{3}, n_{4}\right\}=k}} 1=4 .
$$

Therefore, denoting $q=p^{1-s}$, the inner sum of $H_{p}(s)$ on $m$ is

$$
\begin{aligned}
\left(\sum_{m=0}^{\infty} \frac{H(k, m)}{p^{m(s-1)}}-\sum_{m=0}^{k} \frac{H(k, m)}{p^{m(s-1)}}\right)-\left(\sum_{m=0}^{\infty} \frac{H(k-1, m)}{p^{m(s-1)}}-\sum_{m=0}^{k} \frac{H(k-1, m)}{p^{m(s-1)}}\right) \\
=\left(\left(\frac{1-q^{k+1}}{1-q}\right)^{4}-\sum_{m=0}^{k}\left(\begin{array}{c}
m+3 \\
3
\end{array}\right) q^{m}\right) \\
-\left(\left(\frac{1-q^{k}}{1-q}\right)^{4}-\sum_{m=0}^{k-1}\left(\begin{array}{c}
m+3 \\
3
\end{array}\right) q^{m}-H(k-1, k) q^{k}\right) \\
=\left(\frac{1-q^{k+1}}{1-q}\right)^{4}-\left(\frac{1-q^{k}}{1-q}\right)^{4}-4 q^{k} .
\end{aligned}
$$

Replacing $q$ by $p^{1-s}$ we obtain that

$$
\begin{aligned}
H_{p}(s)= & 1+\left(1-\frac{4}{p(p+3)}\right) \sum_{k=1}^{\infty} p^{k(s-2)} \\
& \times\left(\left(\frac{1-p^{(1-s)(k+1)}}{1-p^{1-s}}\right)^{4}-\left(\frac{1-p^{(1-s) k}}{1-p^{1-s}}\right)^{4}-4 p^{(1-s) k}\right) \\
= & 1+\frac{(p-1)(p+4)}{p(p+3)}\left\{-\frac{4}{p-1}+\sum_{k=1}^{\infty} \frac{\left(1-p^{(1-s)(k+1)}\right)^{4}-\left(1-p^{(1-s) k}\right)^{4}}{\left(1-p^{1-s}\right) p^{k(2-s)}}\right\} .
\end{aligned}
$$

Since

$$
\psi=\mu * \mu * \mu * \mu * I_{d} * B
$$

it is clear that

$$
\sum_{n=1}^{\infty} \frac{\psi(n)}{n^{s}}=\frac{\zeta(s-1)}{\zeta^{4}(s)} \prod_{p \text { prime }} H_{p}(s) .
$$

This completes the proof of Lemma 3, and therefore also the proof of Theorem 1. 


\section{References}

[1] M. V. Berry, V. Tabor, Level clustering in the regular spectrum, Proc. Royal Soc. London A 356 (1997), 375-394.

[2] P. M. Bleher, The energy level spacing for two harmonic oscillators with golden mean ratio of frequencies, J. Stat. Phys. 61 (1990), 869-876.

[3] P. M. Bleher, The energy level spacing for two harmonic oscillators with generic ratio of frequencies, J. Stat. Phys. 63 (1991), 261-283.

[4] F. Boca, C. Cobeli, A. Zaharescu, Distribution of lattice points visible from the origin, Comm. Math. Phys. 213 (2000), no. 2, 433-470.

[5] F. Boca, C. Cobeli, A. Zaharescu, A conjecture of R. R. Hall on Farey points, J. reine angew. Math. 535 (2001), 207-236.

[6] F. Boca, A. Zaharescu, Pair correlation of values of rational functions $(\bmod p)$, Duke Math. J. 105 (2000), no. 2, 267-307.

[7] F. Boca, A. Zaharescu, On the pair correlation for fractional parts of vector sequences, Arch. Math. (Basel) 77 (2001), no. 6, 498-507.

[8] F. P. Boca, A. Zaharescu, The Correlations of Farey Fractions, J. London Math. Soc. (2) 72 (2005), $25-39$.

[9] C. Cobeli, A. Zaharescu, On the distribution of primitive roots $(\bmod p)$, Acta Arith. 83 (1998), $143-153$.

[10] P. Deligne, Séminaire Géometrie Algébrique 4 $\frac{1}{2}$, Lect. Notes Math. 569 (1977), 221-228.

[11] F. J. Dyson, Statistical theory of the energy levels of complex systems, I, J. Math. Phys. 3 (1962), $140-156$.

[12] F. J. Dyson, Statistical theory of the energy levels of complex systems, II, J. Math. Phys. 3 (1962), $157-165$.

[13] F. J. Dyson, Statistical theory of the energy levels of complex systems, III, J. Math. Phys. 3 (1962), $166-175$.

[14] N. D. Elkies, C. T. McMullen, Gaps in $\sqrt{n} \bmod 1$ and ergodic theory, Duke Math. J. 123 (2004), no. 1, 95-139.

[15] T. Estermann, On Kloosterman's sums, Mathematika 8 (1961), 83-86.

[16] J. Friedlander, H. Iwaniec, The Brun-Titchmarsh theorem, Analytic number theory (Kyoto 1996), London Math. Soc. Lect. Note Ser. 247, Cambridge Univ. Press, Cambridge (1997), 85-93.

[17] P. X. Gallagher, On the distribution of primes in short intervals, Mathematika 23 (1976), 4-9.

[18] G. H. Hardy, E. M. Wright, An Introduction to the Theory of Numbers, Clarendon Press, Oxford 1938 (fourth edition 1960).

[19] C. Hooley, An asymptotic formula in the theory of numbers, Proc. London Math. Soc. 7 (1957), $396-413$.

[20] C. Hooley, On the intervals between consecutive terms of sequences, Proc. Symp. Pure Math. 24 (1973), 129-140.

[21] A. A. Karatsuba, Distribution of inverse values in a residue ring modulo a given number (Russian), Dokl. Akad. Nauk 333 (1993), no. 2, 138-139; translation: Russian Acad. Sci. Dokl. Math. 48 (1994), no. 3, 452-454.

[22] A. A. Karatsuba, Fractional parts of functions of a special form, Izv. Ross. Akad. Nauk Ser. Mat. 59 (1995), no. 4, 61-80; translation: Izv. Math. 59 (1995), no. 4, 721-740.

[23] A. A. Karatsuba, Analogues of Kloosterman sums, Izv. Ross. Akad. Nauk Ser. Mat. 59 (1995), no. 5, $93-$ 102; translation: Izv. Math. 59 (1995), no. 5, 971-981.

[24] N. Katz, P. Sarnak, Zeroes of zeta functions and symmetry, Bull. Amer. Math. Soc. (N.S.) 36 (1999), no. 1, $1-26$.

[25] N. Katz, P. Sarnak, Random matrices, Frobenius eigenvalues, and monodromy, Amer. Math. Soc. Colloqu. Publ. 45 (1999).

[26] P. Kurlberg, The distribution of spacings between quadratic residues, II, Israel J. Math. 120 (2000), part A, 205-224.

[27] P. Kurlberg, Z. Rudnick, The distribution of spacings between quadratic residues, Duke Math. J. 100 (1999), no. 2, 211-242.

[28] J. Marklof, Pair correlation densities of inhomogeneous quadratic forms, Ann. Math. (2) 158 (2003), no. 2, 419-471.

[29] J. Marklof, Pair correlation densities of inhomogeneous quadratic forms, II, Duke Math. J. 115 (2002), no. 3, 409-434.

[30] H. L. Montgomery, The pair correlation of zeros of the zeta function, Analytic number theory, Proc. Sympos. Pure Math. XXIV, St. Louis Univ., St. Louis, Mo. (1972), 181-193.

[31] H. L. Montgomery, Ten lectures on the interface between analytic number theory and harmonic analysis, CBMS Reg. Conf. Ser. Math. 84 (1994).

[32] A. Pandey, O. Bohigas, M. J. Giannoni, Level repulsion in the spectrum of two-dimensional harmonic oscillators, J. Phys. A 22 (1989), 4083-4088. 
[33] Z. Rudnick, P. Sarnak, Zeros of principal $L$-functions and random matrix theory, A celebration of John F. Nash, Jr., Duke Math. J. 81 (1996), no. 2, 269-322.

[34] Z. Rudnick, P. Sarnak, The pair correlation function of fractional parts of polynomials, Comm. Math. Phys. 194 (1998), no. 1, 61-70.

[35] Z. Rudnick, P. Sarnak, A. Zaharescu, The distribution of spacings between the fractional parts of $n^{2} \alpha$, Invent. Math. 145 (2001), no. 1, 37-57.

[36] Z. Rudnick, A. Zaharescu, A metric result on the pair correlation of fractional parts of sequences, Acta Arith. 89 (1999), no. 3, 283-293.

[37] Z. Rudnick, A. Zaharescu, The distribution of spacings between small powers of a primitive root, Israel $\mathbf{J}$. Math. 120 (2000), part A, 271-287.

[38] Z. Rudnick, A. Zaharescu, The distribution of spacings between fractional parts of lacunary sequences, Forum Math. 14 (2002), no. 5, 691-712.

[39] P. Sarnak, Values at integers of binary quadratic forms, Harmonic analysis and number theory (Montreal, PQ, 1996), CMS Conf. Proc. 21, Amer. Math. Soc., Providence, RI (1997), 181-203.

[40] V. Sós, On the distribution mod 1 of the sequence $n \alpha$, Ann. Univ. Sci. Budapest, Eötvös Sect. Math. 1 (1958), 127-134.

[41] S. Swierczkowski, On successive settings of an arc on the circumference of a circle, Fundam. Math. 46 (1958), 187-189.

[42] J. M. Vanderkam, Pair correlation of four-dimensional flat tori, Duke Math. J. 97 (1999), 413-438.

[43] J. M. Vanderkam, Values at integers of homogeneous polynomials, Duke Math. J. 97 (1999), $379-412$.

[44] J. M. Vanderkam, Correlations of eigenvalues on multi-dimensional flat tori, Comm. Math. Phys. 210 (2000), 203-223.

[45] A. Weil, On some exponential sums, Proc. Nat. Acad. Sci. USA 34 (1948), 204-207.

[46] H. Weyl, Über die Gleichverteilung von Zahlen mod eins, Math. Ann. 77 (1916), no. 3, 313-352.

[47] E. Wigner, Random matrices in physics, SIAM Rev. 9 (1967), 1-23.

[48] A. Zaharescu, Averages of short exponential sums, Acta Arith. 88 (1999), no. 3, 223-231.

[49] A. Zaharescu, Averages of short exponential sums, II, Acta Arith. 100 (2001), no. 4, 339-348.

[50] A. Zaharescu, Correlation of fractional parts of $n^{2} \alpha$, Forum Math. 15 (2003), no. 1, 1-21.

Department of Mathematics, Koc University, Rumelifeneri Yolu, 34450, Sariyer, Istanbul, Turkey e-mail: ealkan@ku.edu.tr

Department of Mathematics, Eberly College of Science, Pennsylvania State University, McAllister Building, University Park, PA 16802, USA e-mail: xiong@math.psu.edu

Institute of Mathematics of the Romanian Academy, P.O. Box 1-764, 70700 Bucharest, Romania Department of Mathematics, University of Illinois at Urbana-Champaign, Altgeld Hall, 1409 W. Green Street, Urbana, IL 61801, USA

e-mail: zaharesc@math.uiuc.edu

Eingegangen 19. Januar 2007, in revidierter Fassung 2. Juli 2008 